\title{
CUBIC CRITICAL PORTRAITS AND POLYNOMIALS WITH WANDERING GAPS
}

\author{
ALEXANDER BLOKH, CLINTON CURRY, AND LEX OVERSTEEGEN
}

\begin{abstract}
Thurston introduced $\sigma_{d}$-invariant laminations (where $\sigma_{d}(z)$ coincides with $z^{d}: \mathbb{S}^{1} \rightarrow \mathbb{S}^{1}, d \geq 2$ ) and defined wandering $k$-gons as sets $\mathbf{T} \subset \mathbb{S}^{1}$ such that $\sigma_{d}^{n}(\mathbf{T})$ consists of $k \geq 3$ distinct points for all $n \geq 0$ and the convex hulls of all the sets $\sigma_{d}^{n}(\mathbf{T})$ in the plane are pairwise disjoint. He proved that $\sigma_{2}$ has no wandering $k$-gons.

Call a lamination with wandering $k$-gons a WT-lamination. In a recent paper it was shown that uncountably many cubic WT-laminations, with pairwise non-conjugate induced maps on the corresponding quotient spaces $J$, are realizable as cubic polynomials on their (locally connected) Julia sets. Here we use a new approach to construct cubic WTlaminations with the above properties so that any wandering branch point of $J$ has a dense orbit in each subarc of $J$ (we call such orbits condense), and show that critical portraits corresponding to such laminations are dense in the space $\mathcal{A}_{3}$ of all cubic critical portraits.
\end{abstract}

\section{INTRODUCTION}

1.1. Preliminaries. Let $\mathbb{C}$ be the complex plane and $\widehat{\mathbb{C}}=\mathbb{C} \cup\{\infty\}$ be the complex sphere. Theorem 1.1.1 is a special case of a theorem of Thurston [17, Theorem II.5.2].

Theorem 1.1.1 (No wandering vertices for quadratics). Let $P(z)=z^{2}+c$ be a polynomial which has connected Julia set $J_{P}$. Then, if $z_{0} \in J_{P}$ is a point such that $J_{P} \backslash\left\{z_{0}\right\}$ has at least three components, then $z_{0}$ is either preperiodic or eventually maps to the critical point 0 .

A point $z_{0} \in J_{P}$ is called a vertex if $J_{P} \backslash\left\{z_{0}\right\}$ has at least three components, and a vertex is called wandering if it is not periodic and never maps to a critical point. It is shown in [4, Theorem 1.1] that there exist uncountably many cubic polynomials, each of which has a locally connected Julia

Date: March 9, 2010; revised versions December 12, 2010, July 20, 2011 and December 14, 2011.

Key words and phrases. Complex dynamics, locally connected, Julia set, lamination.

The authors were partially supported by NSF grant DMS-0901038 (A.B.) and by NSF grant DMS-0906316 (L.O.). 
set and a wandering vertex. Here we improve on these examples by constructing uncountably many cubic polynomials with locally connected Julia sets whose wandering vertices have condense orbits, where a set $A \subset X$ is called condense if $A$ intersects every non-degenerate subcontinuum of $X$. This is much stronger than the density of the orbit of $z_{0}$ in $J$ (e.g., most subcontinua of dendritic Julia sets are nowhere dense); see [2] for some consequences of having a condense orbit. To state our results precisely, we first briefly describe Thurston's theory of invariant laminations.

Laminations were introduced by Thurston [17, Definition II.4.2] as a tool for studying individual complex polynomials $P: \widehat{\mathbb{C}} \rightarrow \widehat{\mathbb{C}}$ and their parameter space. Let $P$ be a degree $d$ polynomial with a connected Julia set $J_{P}$. Its filled-in Julia set $K_{P}$ is compact, connected, and full, so its complement $\widehat{\mathbb{C}} \backslash K_{P}$ is conformally isomorphic to the open unit disk $\mathbb{D}$. By [13, Theorem 9.5] one can choose a conformal isomorphism $\Psi: \mathbb{D} \rightarrow \widehat{\mathbb{C}} \backslash K_{P}$ so that $\Psi$ satisfies $\Psi\left(z^{d}\right)=\left(\left.P\right|_{\widehat{\mathbb{C}} \backslash K_{P}} \circ \Psi\right)(z)$ for all $z \in \mathbb{D}$. For a locally connected Julia set $J_{P}$, the map $\Psi$ extends to a continuous map $\bar{\Psi}: \overline{\mathbb{D}} \rightarrow \overline{\widehat{\mathbb{C}} \backslash K_{P}}$ [13, Theorem 17.14], semiconjugating $z \mapsto z^{d}$ on $\overline{\mathbb{D}}$ to $\left.P\right|_{\widehat{\mathbb{C}} \backslash K_{P}}$. Let $\psi$ : $\mathbb{S}^{1} \rightarrow J_{P}$ denote the restriction $\left.\bar{\Psi}\right|_{\mathbb{S}^{1}}$, and let $\sigma_{d}: \mathbb{S}^{1} \rightarrow \mathbb{S}^{1}$ denote the map $z \mapsto z^{d}$. Define an equivalence relation $\sim_{P}$ on $\mathbb{S}^{1}$ so that $x \sim_{P} y$ if and only if $\psi(x)=\psi(y)$, and call it the $\sigma_{d}$-invariant lamination generated by $P$. The quotient space $\mathbb{S}^{1} / \sim_{P}=J_{\sim_{P}}$ is homeomorphic to $J_{P}$ and the induced map $f_{\sim_{P}}: J_{\sim_{P}} \rightarrow J_{\sim_{P}}$ defined by $f_{\sim_{P}}=\psi \circ \sigma_{d} \circ \psi^{-1}$ is conjugate to $\left.P\right|_{J_{P}}$. One can introduce abstract laminations (frequently denoted by $\sim$ ) as equivalence relations on $\mathbb{S}^{1}$ similar to laminations generated by polynomials as above (see Section 2). We call $J_{\sim}=\mathbb{S}^{1} / \sim$ a topological Julia set, and denote the map induced by $\sigma_{d}$ on $J_{\sim}$ by $f_{\sim}$.

Laminations are also used in studying the space $\mathcal{P}_{d} \cong \mathbb{C}^{d-1}$ of degree $d \geq 2$ monic centered polynomials $z \mapsto z^{d}+a_{d-2} z^{d-2}+\cdots+a_{0}$. The connectedness locus of $\mathcal{P}_{d}$ is the set $\mathcal{C}_{d}$ of parameters in $\mathcal{P}_{d}$ for which the Julia set is connected (which is a continuum by [5, Corollary 3.7] and [12]). The set $\mathcal{C}_{2}$ is called the Mandelbrot set and is denoted by $\mathcal{M}$. Thurston [17, Definition II.6.9] defined a "meta-lamination", referred to as QML, and showed that the closure of the space of all $\sigma_{2}$-invariant (quadratic) laminations can be thought of as the quotient space $\mathbb{S}^{1} / \mathrm{QML}=\mathcal{M}_{c}$, a locally connected continuum that serves as a combinatorial model of the boundary of $\mathcal{M}$. The exact relationship between $\mathcal{M}_{c}$ and $\mathcal{M}$ is unknown. Thurston conjectured that the boundary of $\mathcal{M}$ is homeomorphic to $\mathcal{M}_{c}$, which, if true, would imply that $\mathcal{M}$ is locally connected. A crucial role in his study is played by the next theorem. 
No Wandering Triangles Theorem ([17, Theorem II.5.2]). Let $\sim$ be $a \sigma_{2}$ invariant lamination. If $\mathrm{g}$ is a class of cardinality at least three, then $\mathrm{g}$ is either eventually critical or eventually periodic.

Thus, branch points in degree 2 topological Julia sets either are precritical or preperiodic, which can be regarded as a generalization of the corresponding property of continuous maps of finite graphs. Also, it follows from the No Wandering Triangles Theorem that branch points of $\mathcal{M}_{c}$ correspond to laminations with preperiodic critical classes. This makes the problem of extending Thurston's result to higher degrees, posed by Thurston in [17], important. Indeed, J. Kiwi [8, Theorem 1.1] answers this call by showing that a wandering non-precritical gap in a $\sigma_{d}$-invariant lamination is at most a $d$-gon. Thus all infinite $\sim$-classes and Jordan curves in $J_{\sim}$ are preperiodic. Later on [3, Theorem B] it was shown that if $\Gamma$ is a non-empty collection of wandering non-precritical $d_{j}$-gons $(j=1,2, \ldots)$ of a $\sigma_{d}$-invariant lamination with distinct grand orbits, then $\sum_{\Gamma}\left(d_{j}-2\right) \leq d-2$. Thus, there are bounds on the number of wandering gaps with distinct grand orbits.

However, even for $\sigma_{3}$-invariant (henceforth cubic) laminations wandering triangles exist: by [4], Theorem 1.1, there are uncountably many pairwise non-conjugate cubic polynomials $P$ which have dendritic Julia sets with a wandering branch point. Hence for each such polynomial $P$ the corresponding lamination $\sim_{P}$ has a wandering triangle. Since the construction in [4] is quite specific, the corresponding "wandering" dynamics might be rare.

This paper gives a more general and flexible construction than that in [4], extending the above. Let $\mathcal{A}_{3}$ be the space of all cubic critical portraits. We construct cubic laminations such that the forward orbit of a wandering triangle is dense in the entire lamination (this is much stronger than in [4]), and prove that their critical portraits form a locally uncountable and dense subset of $\mathcal{A}_{3}$. Thus, critical portraits with wandering vertices in their Julia sets are not rare. Even more, the topological polynomial of each of these laminations is conjugate to a polynomial restricted to its Julia set by [2].

In conclusion we want to thank the referees for careful reading of the manuscript and useful remarks which helped us improve the paper.

1.2. Statement of the results. We parameterize the circle as $\mathbb{S}^{1}=\mathbb{R} / \mathbb{Z}$ with total arclength 1 . In this parameterization the map $\sigma_{d}$ corresponds to the map $t \mapsto d \cdot t \bmod 1$. The positive direction on $\mathbb{S}^{1}$ is counterclockwise, and by an $\operatorname{arc}(p, q)$ in $\mathbb{S}^{1}$ we mean the positively oriented arc from $p$ to $q$. A monotone map $g:(p, q) \rightarrow \mathbb{S}^{1}$ is a map such that for each $y \in \mathbb{S}^{1}$ the set $g^{-1}(y)$ is connected. A monotone map is called strictly monotone if it is one-to-one. Given a set $A \subset \mathbb{S}^{1}$, we denote the cardinality of $A$ by $|A|$ and the convex hull of $A$ in the closed unit disk by $\mathrm{Ch}(A)$ (for our purposes it 
does not matter whether we use the Euclidian or the hyperbolic metric). In what follows, given a map $g$, a ( $g$-)image of a set $A$ is the image of $A$ under an iterate of $g$ while the first ( $g$-)image of a set $A$ is the set $g(A)$. Similar language will be used for preimages.

Given an equivalence relation $\sim$ on $\mathbb{S}^{1}$, the equivalence classes are called $(\sim-)$ classes and are denoted by boldface letters. A $\sim$-class consisting of two points is called a leaf; a class consisting of at least three points is called a gap (this is more restrictive than Thurston's definition in [17]).

Fix an integer $d>1$. Then an equivalence relation $\sim$ is said to be a $\sigma_{d}$-invariant lamination if:

(1) $\sim$ is closed: the graph of $\sim$ is a closed set in $\mathbb{S}^{1} \times \mathbb{S}^{1}$;

(2) $\sim$-classes are pairwise unlinked: if $\mathbf{g}_{1}$ and $\mathbf{g}_{2}$ are distinct $\sim$-classes, their convex hulls $\mathrm{Ch}\left(\mathbf{g}_{1}\right), \mathrm{Ch}\left(\mathbf{g}_{2}\right)$ in the closed unit disk $\overline{\mathbb{D}}$ are disjoint;

(3) $\sim$-classes are totally disconnected (and hence $\sim$ has uncountably many classes) provided $\mathbb{S}^{1}$ is not one class;

(4) $\sim$ is forward invariant: for a class $\mathbf{g}$, the set $\sigma_{d}(\mathbf{g})$ is also a class;

(5) $\sim$ is backward invariant: for a class $\mathbf{g}$, its first preimage $\sigma_{d}^{-1}(\mathbf{g})=$ $\left\{x \in \mathbb{S}^{1}: \sigma_{d}(x) \in \mathbf{g}\right\}$ is a union of classes; and

(6) $\sim$ is gap invariant: for any gap $\mathbf{g}$, the map $\left.\sigma_{d}\right|_{\mathbf{g}}: \mathbf{g} \rightarrow \sigma_{d}(\mathbf{g})$ is a covering map with positive orientation, i.e., for every connected component $(s, t)$ of $\mathbb{S}^{1} \backslash \mathbf{g}$ the arc $\left(\sigma_{d}(s), \sigma_{d}(t)\right)$ is a connected component of $\mathbb{S}^{1} \backslash \sigma_{d}(\mathbf{g})$.

Notice that (3) and (5) are implied by (4).

Call a class $\mathbf{g}$ critical if $\left.\sigma_{d}\right|_{\mathbf{g}}: \mathbf{g} \rightarrow \sigma_{d}(\mathbf{g})$ is not one-to-one, and precritical if $\sigma_{d}^{j}(\mathbf{g})$ is critical for some $j \geq 0$. Call $\mathbf{g}$ preperiodic if $\sigma_{d}^{i}(\mathbf{g})=\sigma_{d}^{j}(\mathbf{g})$ for some $0 \leq i<j$. A gap $\mathbf{g}$ is wandering if $\mathbf{g}$ is neither preperiodic nor precritical, and a lamination which has a wandering gap is called a $W T$ lamination. For a lamination $\sim$, let $J_{\sim}=\mathbb{S}^{1} / \sim$, and let $\pi_{\sim}: \mathbb{S}^{1} \rightarrow J_{\sim}$ be the corresponding quotient map. Then the map $f_{\sim}: J_{\sim} \rightarrow J_{\sim}$ defined by $f_{\sim}=\pi_{\sim} \circ \sigma_{d} \circ \pi_{\sim}^{-1}$ is the map induced on $J_{\sim}$ by $\sigma_{d}$ (the map $f_{\sim}$ is well-defined in view of (4)). We call $f_{\sim}$ a topological polynomial, and $J_{\sim}$ a topological Julia set.

Though we define laminations as specific equivalence relations on $\mathbb{S}^{1}$, one can also work with a corresponding collection of chords, called a geometric $\left(\sigma_{d}\right.$-invariant) lamination. Given a $\sigma_{d}$-invariant lamination $\sim$, its geometric lamination $\left(\mathcal{L}_{\sim}, \sigma_{3}\right)$ is defined as the union of all chords in the boundaries of convex hulls of all $\sim$-classes; the map $\sigma_{3}$ is then extended over $\mathcal{L}_{\sim}$ by linearly mapping each chord in $\mathcal{L}_{\sim}$ forward. Clearly, $\mathcal{L}_{\sim}$ is a 
closed family of chords of $\overline{\mathbb{D}}$ (in the above situation we also include degenerate $\sim$-classes in the list of chords). Geometric laminations have properties similar to the properties of the laminations introduced by Thurston [17, Definition II.4.2]. One of the main ideas of this paper is to study finite truncations of geometric laminations $\left(\mathcal{L}_{\sim}, \sigma_{3}\right)$ defined up to an order preserving conjugacy and considering such finite laminations as purely combinatorial objects.

1.2.1. Critical portraits. Fix $d \geq 2$. A key tool in studying $\mathcal{C}_{d}$ is critical portraits, introduced in [6], and widely used afterward [1, 7, 10, 14, 15]. We now recall some standard material. Here we follow [10, Section 3] closely. Call a chord of the circle, with endpoints $a, b \in \mathbb{S}^{1}$, critical if $\sigma_{d}(a)=\sigma_{d}(b)$.

Definition 1.2.1. Fix $d \geq 2$. A $\left(\sigma_{d^{-}}\right)$critical portrait is a collection $\Theta=$ $\left\{\Theta_{1}, \ldots, \Theta_{n}\right\}$ of finite subsets of $\mathbb{S}^{1}$ such that the following hold.

(1) The boundary of the convex hull $\mathrm{Ch}\left(\Theta_{i}\right)$ of each set $\Theta_{i}$ consists of critical chords;

(2) the sets $\Theta_{1}, \ldots, \Theta_{n}$ are pairwise unlinked (that is, their convex hulls are pairwise disjoint); and

(3) $\sum\left(\left|\Theta_{i}\right|-1\right)=d-1$.

To comment on Definition 1.2.1 we need the following terminology: the sets $\Theta_{1}, \ldots, \Theta_{n}$ are called the initial sets of $\Theta$ (or $\Theta$-initial sets). The convex hulls of the $\Theta$-initial sets divide the rest of the unit disk $\mathbb{D}$ into components. Consider one such component, say $U$. Then $\partial U$ consists of circular arcs and critical chords $\ell_{1}, \ldots, \ell_{k}$. If we extend $\sigma_{d}$ linearly on the chords in the boundary of $U$, then, by Definition 1.2.1, $\partial U$ maps onto $\mathbb{S}^{1}$ one-to-one except for the collapsing critical chords $\ell_{1}, \ldots, \ell_{k}$. In fact, Definition 1.2.1 is designed to achieve this dynamical property related to situations of the following kind. Suppose that $P$ is a polynomial of degree $d$ with dendritic (i.e., locally connected and containing no simple closed curve) Julia set $J_{P}$ such that all its critical values are endpoints of $J_{P}$. Then the arguments of rays landing at the critical points of $P$ form the initial sets of a certain critical portrait associated with this polynomial.

Let $\Theta$ be a critical portrait. Denote by $A(\Theta)$ the union of all angles from the initial sets of $\Theta$. As was remarked above, the convex hulls of the $\Theta$ initial sets divide the rest of the unit disk $\mathbb{D}$ into components. According to Definition 1.2.2, points of $\mathbb{S}^{1} \backslash A(\Theta)$ belonging to the boundary of one such component will be declared equivalent. However, since we need this notion in more general situations, we will not assume in Definition 1.2.2 that $\Theta$ is a critical portrait. 
Definition 1.2.2 ([1], [6], [7], [10, Definition 3.4]). Let $\Theta=\left\{\Theta_{1}, \ldots, \Theta_{n}\right\}$ be a finite collection of pairwise unlinked finite subsets of $\mathbb{S}^{1}$ (not necessarily a critical portrait). Angles $\alpha, \beta \in \mathbb{S}^{1} \backslash A(\Theta)$ are $\Theta$-unlinked equivalent if $\{\alpha, \beta\}, \Theta_{1}, \ldots, \Theta_{n}$ are pairwise unlinked (i.e., if the chord $\overline{\alpha \beta}$ is disjoint from the $\left.\bigcup_{i=1}^{k} \operatorname{Ch}\left(\Theta_{k}\right)\right)$. The classes of equivalence $L_{1}(\Theta), \ldots, L_{d}(\Theta)$ are called $\Theta$-unlinked classes. Each $\Theta$-unlinked class $L$ is the intersection of $\mathbb{S}^{1} \backslash A(\Theta)$ with the boundary of a component of $\mathbb{D} \backslash \bigcup \operatorname{Ch}\left(\Theta_{i}\right)$. If $\Theta$ is a degree $d$ critical portrait, then each $\Theta$-unlinked class of $\Theta$ is the union of finitely many open (in $\mathbb{S}^{1}$ ) arcs of total length $1 / d$. Thus, in this case there are precisely $d \Theta$-unlinked classes.

We introduce the following topology on the set of critical portraits.

Definition 1.2.3 (compact-unlinked topology [10, Definition 3.5]). Define the space $\mathcal{A}_{d}$ as the set of all degree $d$ critical portraits. We endow it with the compact-unlinked topology: the coarsest topology on $\mathcal{A}_{d}$ such that, for any compact set $X \subset \mathbb{S}^{1}$, the set of critical portraits whose critical leaves are unlinked with $X$ is open.

For example, $\mathcal{A}_{2}$ is the quotient of the circle with antipodal points identified, so it is homeomorphic to $\mathbb{S}^{1}$. As another example, take a cubic critical portrait which consists of a triangle $T$ with vertices $a, b, c$ and three critical edges, and choose compact sets $X_{1}, X_{2}$, and $X_{3}$ in the three components of $\mathbb{S}^{1} \backslash\{a, b, c\}$. Then every neighborhood of $T$ includes critical triangles close to $T$ and pairs of disjoint critical leaves each of which is close to an edge of $T$.

For a critical portrait $\Theta$, a lamination $\sim$ is called $\Theta$-compatible if all $\Theta$ initial sets are subsets of $\sim$-classes. The trivial lamination which identifies all points of the circle is compatible with any critical portrait. If there is a $\Theta$-compatible WT-lamination, $\Theta$ is called a critical WT-portrait.

An important tool for describing the dynamics of a lamination is the itinerary. For simplicity and because it suits our purpose, the following is defined for critical portraits consisting of $d-1$ disjoint critical leaves. For a critical portrait $\Theta$ and a point $t \in \mathbb{S}^{1}$, we define two types of itineraries: one-sided itineraries, denoted $\operatorname{itin}^{+}(t)$ and $\operatorname{itin}^{-}(t)$ which are sequences of $\Theta$-unlinked classes (see [10, Definition 3.13]), and the itinerary itin $(t)$ corresponding to the Markov partition into $\Theta$-unlinked classes and elements of $\Theta$. We define $\operatorname{itin}^{+}(t)=i_{0}^{+} i_{1}^{+} i_{3}^{+} \ldots$ where $i_{k}^{+}$is the $\Theta$-unlinked class which contains the interval $\left(\sigma_{d}^{k}(t), \sigma_{d}^{k}(t)+\epsilon\right)$ for some small $\epsilon>0$; $\operatorname{itin}^{-}(t)=i_{0}^{-} i_{1}^{-} \ldots$ is similarly defined. We define $\operatorname{itin}(t)=i_{0} i_{1} i_{2}, \ldots$ such that $i_{k}$ is the $\Theta$-unlinked class or critical leaf containing $\sigma_{d}^{k}(t)$. Thus, if $\sigma_{d}^{k}(t)$ is not an endpoint of a critical leaf, then $i_{k}, i_{k}^{+}$, and $i_{k}^{-}$are all equal, and if $\sigma_{d}^{k}(t)$ is an endpoint of a critical leaf, then $i_{k}, i_{k}^{+}$, and $i_{k}^{-}$are all different. 
An important class of critical portraits are critical portraits with aperiodic kneading. A critical portrait $\Theta$ with $d-1$ critical leaves has aperiodic kneading if for each angle $\theta \in A(\Theta)$ the itineraries $\operatorname{itin}^{+}(\theta)$ and $\operatorname{itin}^{-}(\theta)$ are not periodic [10, Definition 4.6]. The family of all critical portraits with $d-1$ critical leaves and aperiodic kneading sequence is denoted by $\mathcal{A} \mathcal{P}_{d}$.

Definition 1.2.4 (Definition 4.5 [10]). Let $\Theta$ be a critical portrait with aperiodic kneading. The lamination $\sim_{\Theta}$ is defined as the smallest equivalence relation such that if $\operatorname{itin}^{+}(x)=\operatorname{itin}^{-}(y)$ then $x$ and $y$ are $\sim_{\Theta}$-equivalent; it is said to be the lamination generated by $\Theta$.

Now we quote a fundamental result of Kiwi [10] (see also [9]). To state it we need the following definitions. A map $\varphi: X \rightarrow Y$ from a continuum $X$ to a continuum $Y$ is said to be monotone if $\varphi$-preimages of points are continua. A dendrite is a locally connected non-degenerate continuum which contains no subsets homeomorphic to $\mathbb{S}^{1}$.

Theorem 1.2.5 ([9, 10]). Let $\Theta \in \mathcal{A} \mathcal{P}_{d}$. Then the lamination $\sim_{\Theta}$ is the unique $\Theta$-compatible $\sigma_{d^{-}}$-invariant lamination, $J_{\sim_{\Theta}}$ is a dendrite, all $\sim_{\Theta^{-}}$ classes are finite, and there exists a polynomial $P$ whose Julia set $J_{P}$ is a non-separating continuum in the plane such that $\left.P\right|_{J_{P}}$ is semiconjugate to $\left.f_{\sim_{\Theta}}\right|_{J_{\sim_{\Theta}}}$ by a monotone map $\psi: J_{P} \rightarrow J_{\sim_{\Theta}}$. For each P-preperiodic point $x \in J_{P}$ the set $\psi^{-1}(\psi(x))=\{x\}$. Furthermore, $J_{P}$ is locally connected at preperiodic points.

Remark 1.2.6. This theorem is an amalgamation of several results of Kiwi; we will describe those results here. Suppose that $\Theta$ satisfies the hypothesis. Then, by [10, Corollary 3.26], there is a polynomial $P$ with connected Julia set whose critical portrait is $\Theta$. By [10, Theorem 1], all cycles of $P$ are repelling, and the real lamination of $P$ is the unique $\Theta$-compatible lamination. By [9, Theorem 5.12], $\left.P\right|_{J_{P}}$ is monotonically semiconjugate to $f_{\mid} \sim_{\Theta}$; since $J_{P}$ is non-separating, it follows that $J_{\sim_{\Theta}}$ is a dendrite. That the monotone projection $\psi: J_{P} \rightarrow J_{\sim_{\Theta}}$ is one-to-one at $P$-preperiodic points follows from [9, Theorem 2], and that $J_{P}$ is locally connected at $P$-preperiodic points follows from [9, Corollary 1.2].

1.2.2. Condensity and main results. For a topological space $X$, a set $A \subset$ $X$ is continuum-dense (briefly, condense) in $X$ if $A \cap B \neq \emptyset$ for each nondegenerate continuum $B \subset X$. If $X$ is a dendrite, $A$ is condense in $X$ if and only if $A$ intersects every open arc. A condense set $A$ is dense in $X$, but condensity is stronger than density; for example, the set of endpoints of a non-interval dendritic Julia set $J$ is residual, dense and disjoint from all non-degenerate open $\operatorname{arcs}$ in $J$. If $x$ is a point with condense orbit in the Julia set $J_{P}$ of a polynomial $P$, then $J_{P}$ is a dendrite or a Jordan curve [2, Theorem 1.4]. 
Let $\mathcal{W} \mathcal{T}_{3}$ be the family of all cubic critical WT-portraits.

Main theorem. For each open $U \subset \mathcal{A}_{3}$ there is an uncountable set $\mathcal{B} \subset$ $U \cap \mathcal{A P}_{d} \cap \mathcal{W} \mathcal{T}_{3}$ such that the following facts hold:

(1) there exists a wandering branch point in $J_{\sim_{\Theta}}$ whose orbit is condense in $J_{\sim_{\Theta}}$;

(2) the maps $\left\{\left.f_{\sim_{\Theta}}\right|_{J_{\sim_{\Theta}}} \mid \Theta \in \mathcal{B}\right\}$ are pairwise non-conjugate;

(3) for each $\Theta \in \mathcal{B}$ there exists a cubic polynomial $P_{\Theta}$ such that $\left.P_{\Theta}\right|_{J_{P_{\Theta}}}$ is conjugate to $\left.f_{\sim_{\Theta}}\right|_{J_{\sim_{\Theta}}}$.

\section{COMBinAtorial CONSTRUCTION}

Let us recall that by a cubic WT-lamination we mean a $\sigma_{3}$-invariant lamination which admits a wandering (i.e., non-precritical and non-preperiodic) gap. By [3, Theorem B], a wandering gap in such a lamination is a triangle. We construct a cubic WT-lamination by means of a sequence $\left(\left(\mathcal{L}_{i}, g_{i}\right)\right)_{i=1}^{\infty}$ of finite cubic critical laminations, where $\left(\mathcal{L}_{i+1}, g_{i+1}\right)$ continues $\left(\mathcal{L}_{i}, g_{i}\right)$ (the definitions are given below). There is a limit lamination $\mathcal{L}=\bigcup_{i=1}^{\infty} \mathcal{L}_{i}$ with a map $g$ defined on $\bigcup \mathcal{L}=\bigcup_{i=1}^{\infty}\left(\bigcup \mathcal{L}_{i}\right)$ so that $g$ and $g_{i}$ agree on $\mathcal{L}_{i}$ for each $i$. By Theorem 3.3 of [4], $\bigcup \mathcal{L}_{i}$ can be embedded in $\mathbb{S}^{1}$ by means of an order preserving map so that the map induced on its image can be extended to $\sigma_{3}$. This will give rise to the desired invariant cubic WT-lamination.

Unlike [4] (where the construction was rather specific), we are concerned not only with the existence of wandering triangles, but also with how complicated and dense their dynamics can be. In addition, we investigate how common WT-critical portraits are in $\mathcal{A}_{3}$. To address these issues, we develop a construction with new features. Compared to [4] they can be summarized as follows: (1) all preimages of both critical leaves are represented in $\left(\left(\mathcal{L}_{i}, g_{i}\right)\right)_{i=1}^{\infty}$, and the wandering triangle approaches them all (this part is responsible for showing that the corresponding quotient spaces i.e., topological Julia sets - are dendrites with wandering non-precritical branch points whose orbits are condense); (2) the initial segments of the constructed laminations can be chosen arbitrarily close to any given finite lamination (this part is responsible for the density of WT-critical portraits in $\mathcal{A}_{3}$ ).

2.1. Finite laminations. In this section we study finite cubic laminations, in particular finite cubic WT-laminations modeling a $\sigma_{3}$-invariant geometric lamination with a wandering triangle at a finite step of its orbit. For instance, if $\sim$ is a cubic invariant lamination with a wandering triangle $\mathbf{T}_{1}$ and critical leaves $\mathbf{c}$ and $\mathbf{d}$, then a collection consisting of $\mathbf{c}, \mathbf{d}$, finitely many of their images and preimages, and finitely many images of $\mathbf{T}_{1}$, form a finite cubic WT-lamination (where $g$ is the restriction of $\sigma_{3}$ ). 
Definition 2.1.1 (Finite laminations). We will be interested in three levels of specialization for finite laminations.

Finite lamination: A finite lamination is a finite collection $\mathcal{L}$ of finite, pairwise unlinked subsets of $\mathbb{S}^{1}$. The elements of $\mathcal{L}$ are called $\mathcal{L}$ classes, and the union of points of all $\mathcal{L}$-classes is the basis of $\mathcal{L}$. By a sublamination of a finite lamination $\mathcal{L}$ we mean a subcollection of $\mathcal{L}$-classes.

Dynamical lamination: A dynamical lamination is a pair $(\mathcal{L}, g)$ where $\mathcal{L}$ is a finite lamination, $g$ is a map defined on the basis of a finite sublamination $\widehat{\mathcal{L}}$ of $\mathcal{L}$ which can be extended to a covering map of $\mathbb{S}^{1}$ of degree 3 that maps $\widehat{\mathcal{L}}$-classes onto $\mathcal{L}$-classes. Note that the pair $(\mathcal{L}, g)$ determines $\widehat{\mathcal{L}}$ since by definition $\operatorname{Dom}(g)$ is the basis of $\widehat{\mathcal{L}}$. Any class on which $g$ is not defined is called a last class of $\mathcal{L}$.

Critical lamination: A dynamical lamination $(\mathcal{L}, g)$ is critical if there are two-point classes $\mathbf{c}$ and $\mathbf{d}$ of $\mathcal{L}$, called the critical leaves of $\mathcal{L}$, modeling a cubic critical portrait: $g(\mathbf{c})$ and $g(\mathbf{d})$ are points, and every $\{\mathbf{c}, \mathbf{d}\}$-unlinked class, intersected with the domain of $g$, maps forward in an order-preserving fashion.

To establish similarity among finite laminations we need Definition 2.1.2.

Definition 2.1.2. A bijection $h: X \rightarrow Y$ between sets $X, Y \subset \mathbb{S}^{1}$ is an order isomorphism if it preserves circular order. If $A, A^{\prime} \subset \mathbb{S}^{1}$, we say maps $f: A \rightarrow A$ and $g: A^{\prime} \rightarrow A^{\prime}$ are conjugate if they are conjugate in a set-theoretic sense by an order isomorphism $h: A \rightarrow A^{\prime}$. Note that we do not require that an order isomorphism is continuous. Finite (dynamical) laminations $\mathcal{L}$ and $\mathcal{L}^{\prime}$ are order isomorphic if there is a (conjugating, respectively) order isomorphism between $\bigcup \mathcal{L}$ and $\bigcup \mathcal{L}^{\prime}$ that sends $\mathcal{L}$-classes to $\mathcal{L}^{\prime}$-classes.

$\mathcal{L}$-classes could be of three distinct types.

Definition 2.1.3. For a finite lamination $\mathcal{L}$, we refer to $\mathcal{L}$-classes consisting of one point as buds, of two points as leaves, of 3 points as triples (triangles), and to all $\mathcal{L}$-classes consisting of more than 2 points as gaps.

We identify classes with their convex hulls in $\overline{\mathbb{D}}$. The convex hulls of leaves, triples (triangles), and gaps are also called leaves, triples (triangles), and gaps. The boundary chords of a non-degenerate class are called edges. In particularly, every leaf is an edge. We often talk of $\widehat{\mathcal{L}}$ when we really mean $\bigcup \widehat{\mathcal{L}}$ and regard the map $g$ as mapping the convex hull of a class of $\widehat{\mathcal{L}}$ to the convex hull of a class of $\mathcal{L}$. Chords of $\mathbb{D}$ are denoted $\bar{a}, \bar{b}$ etc; a chord with endpoints $x, y$ is denoted by $\overline{x y}$. 
Given an interval $(p, q) \subset \mathbb{S}^{1}$, a strictly monotonically increasing map $g$ : $(p, q) \rightarrow \mathbb{S}^{1}$ is a strictly monotone map which preserves circular orientation. For $A \subset \mathbb{S}^{1}$, a map $f: A \rightarrow f(A)$ is of degree 3 if there are $x_{0}<x_{1}<$ $x_{2}<x_{3}=x_{0}$ with $\left.f\right|_{A \cap\left[x_{i}, x_{i+1}\right)}$ strictly monotonically increasing for all $i=0,1,2$ and there are no two points with the same property.

Definition 2.1.4. A critical lamination $(\mathcal{L}, g)$, which contains a designated triple $\mathbf{T}_{1}$ as a class, is called a finite cubic WT-lamination if:

(1) $(\mathcal{L}, g)$ is of degree 3 ;

(2) $\mathcal{L}$ has a pair of disjoint critical leaves $\mathcal{C}=\{\mathbf{c}, \mathbf{d}\}$;

(3) each class $\mathbf{a} \in \mathcal{L}$ satisfies exactly one of the following:

- $\mathbf{a}$ is a bud, in which case $\mathbf{a}=g^{j}(\mathbf{c})$ for some $j$ or $\mathbf{a}=g^{k}(\mathbf{d})$ for some $k$ (but not both),

- a is a leaf such that $g^{i}(\mathbf{a})$ is a critical leaf for some $i$, or

- $\mathbf{a}$ is a triple such that $\mathbf{a}=g^{n}\left(\mathbf{T}_{1}\right)$ for some $n$

(so leaves in $\mathcal{L}$ are pullbacks of $\mathbf{c}$ or $\mathbf{d}$, buds are images of $\mathbf{c}$ or $\mathbf{d}$, and triangles are images of $\mathbf{T}_{1}$ );

(4) all classes from the grand $g$-orbits of $\mathbf{T}_{1}, \mathbf{c}$, and $\mathbf{d}$ are unlinked.

We will always denote the $n^{\text {th }}$ triple in the orbit of $\mathbf{T}_{1}$ as $\mathbf{T}_{n}=g^{n-1}\left(\mathbf{T}_{1}\right)$. The last classes of $\mathcal{L}$, which are eventual images of $\mathbf{T}_{1}, \mathbf{c}$ and $\mathbf{d}$, are denoted by $\mathbf{T}^{l}(\mathcal{L})=\mathbf{T}^{l}, \mathbf{c}^{\prime}(\mathcal{L})=\mathbf{c}^{\prime}$ and $\mathbf{d}^{\prime}(\mathcal{L})=\mathbf{d}^{\prime}$; we call $\mathbf{T}^{l}$ the last triple of $\mathcal{L}$ (here, the superscript $l$ stands for "last").

Observe that in Definition 2.1.4 we stipulate that no bud of a finite cubic lamination is the common image of both critical leaves. This is because a cubic lamination with a wandering triangle must have two critical leaves with disjoint orbits [3, Theorem A].

2.2. Continuing to a cubic WT-lamination. We will build larger and larger finite laminations, only achieving a true lamination with a wandering triangle in the limit. We say that a dynamical lamination $\left(\mathcal{L}^{\prime}, g^{\prime}\right)$ continues a dynamical lamination $(\mathcal{L}, g)$ if

(1) $\mathcal{L} \subset \mathcal{L}^{\prime}$ and $\widehat{\mathcal{L}} \subset \widehat{\mathcal{L}}^{\prime}$;

(2) $g$ and $g^{\prime}$ have the same degree 3 ; and

(3) $\left.g^{\prime}\right|_{\operatorname{Dom}(g)}=g$.

A natural way to continue a finite lamination uses admissible extensions of the map $g$ to the circle. A covering map $F: \mathbb{S}^{1} \rightarrow \mathbb{S}^{1}$ of degree three such that $\left.F\right|_{\operatorname{Dom}(g)}=g$ is said to be an admissible extension of $g$ if $F$ restricted to any complementary interval of $\operatorname{Dom}(g)=\widehat{\mathcal{L}}$ is strictly monotonically increasing. Let $F$ be an admissible extension of $g$. A forward continuation 
is a continuation such that all elements of $\mathcal{L}^{\prime} \backslash \mathcal{L}$ are forward images of $\mathcal{L}$ classes. If $(\mathcal{L}, g)$ is a finite cubic WT-lamination, then $F$ is a first forwardcontinuing extension of $g$ if $\left(\mathcal{L} \cup\left\{F\left(T^{l}\right)\right\},\left.F\right|_{\widehat{\mathcal{L}} \cup\left\{T^{l}\right\}}\right)=\left(\mathcal{L}_{F},\left.F\right|_{\widehat{\mathcal{L}} \cup\left\{T^{l}\right\}}\right)$ is a dynamical lamination (where $\mathbf{T}^{l}$ is the last triple of $\left.\mathcal{L}\right)$; then $\left(\mathcal{L}_{F},\left.F\right|_{\widehat{\mathcal{L}} \cup\left\{T^{l}\right\}}\right)$ is called the (first) forward continuation of $(\mathcal{L}, g)$ associated to $F$.

A backward continuation is a continuation such that all elements of $\mathcal{L}^{\prime} \backslash \mathcal{L}$ eventually map to $\mathcal{L}$-classes. A natural way to construct backward continuations is to pull classes back under an admissible extension of $g$. Let $F$ be an admissible extension of $g$, let $U$ be a $\widehat{\mathcal{L}}$-unlinked class containing no $\mathcal{L}$-class, and let $\mathrm{x}$ be any $\mathcal{L}$-class contained in $F(U)$. The unambiguous backward continuation of $(\mathcal{L}, g)$ (pulling $\mathbf{x}$ into $U$ under $F$ ) is the backward continuation $\left(\mathcal{L}^{\prime}, g^{\prime}\right)$ such that $\mathcal{L}^{\prime}=\mathcal{L} \cup\left\{\mathbf{x}^{\prime}\right\}$, where $\mathrm{x}^{\prime}$ is the first $F$-preimage of $\mathrm{x}$ in $U$, and

$$
g^{\prime}(t)=\left\{\begin{array}{ll}
g(t) & \text { if } t \in \bigcup \widehat{\mathcal{L}} \\
F(t) & \text { if } t \in \mathbf{x}^{\prime}
\end{array} .\right.
$$

As we prove in the following Lemma, unambiguous backward continuations are combinatorially unique, and therefore may be accomplished without reference to a particular admissible extension. For this reason, we can also iteratively construct unambiguous backward continuations without worry. We will also call such continuations unambiguous backward continuations.

Lemma 2.2.1. Let $(\mathcal{L}, g)$ be a critical lamination, let $U$ be a $\widehat{\mathcal{L}}$-unlinked class, and let $\mathrm{x}$ be a $\mathcal{L}$-class. If $F$ is an admissible extension of $g$ such that $F(U)$ contains $\mathbf{x}$, then the unambiguous extension $\left(\mathcal{L}^{\prime}, g^{\prime}\right)$ pulling $\mathbf{x}$ into $U$ under $F$ is defined and is a critical lamination of the same degree. Further, for any other admissible extensions $F^{\prime}$ of $g$, the backward continuation pulling $\mathrm{x}$ into $U$ under $F^{\prime}$ is defined and isomorphic to $\left(\mathcal{L}^{\prime}, g^{\prime}\right)$.

Proof. Since $\mathcal{L}$ contains two critical leaves and is of degree 3, then $\left.F\right|_{U}$ is one-to-one and order preserving, and the image set $F(U)$ is independent of the particular extension $F$. Construct $\left(\mathcal{L}^{\prime}, g^{\prime}\right)$ as the unambiguous backward continuation pulling $\mathrm{x}$ into $U$ under $F$. Since $\left.F\right|_{U}$ is order-preserving, the resulting lamination $\left(\mathcal{L}^{\prime}, g^{\prime}\right)$ is of the same degree. Further, for any other extension $F^{\prime}$, we see that the corresponding unambiguous continuation $\left(\mathcal{L}^{\prime \prime}, g^{\prime \prime}\right)$ is conjugate to $\left(\mathcal{L}^{\prime}, g^{\prime}\right)$ by the map $f: \bigcup \mathcal{L}^{\prime} \rightarrow \bigcup \mathcal{L}^{\prime \prime}$ defined by

$$
f(t)= \begin{cases}t & \text { if } t \notin \mathbf{x}^{\prime} \\ \left(\left.F^{\prime}\right|_{U} ^{-1} \circ F\right)(t) & \text { if } t \in \mathbf{x}^{\prime}\end{cases}
$$

where $\mathbf{x}^{\prime}$ is the $g^{\prime}$-preimage of $\mathbf{x}$. 


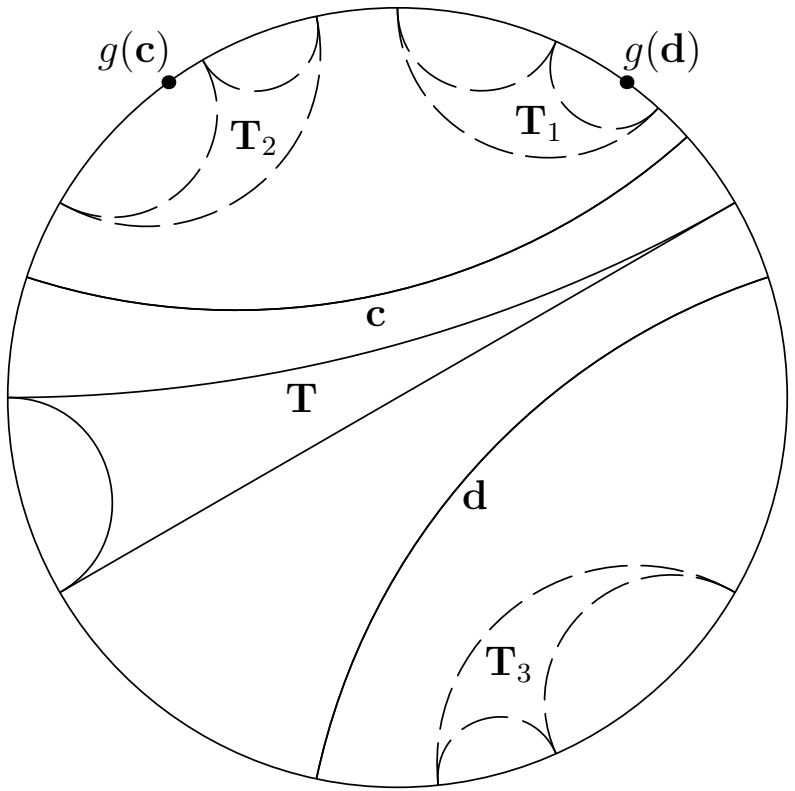

FIGURE 2.2.1. Consider the finite dynamical lamination $\mathcal{L}=\{\mathbf{c}, \mathbf{d}, \mathbf{T}, g(\mathbf{c}), g(\mathbf{d})\}$ pictured here and a few possible backward continuations of $(\mathcal{L}, g)$ whose new elements are pictured in dotted lines. It is evident that all backward continuations of $(\mathcal{L}, g)$ adding only a first preimage of $\mathbf{T}$ under $\mathbf{d}$ (like $\mathbf{T}_{3}$, as illustrated) are order isomorphic. However, the choice of a first preimage of $\mathbf{T}$ in the component of $\mathbb{S}^{1} \backslash \mathbf{c}$, containing the last classes $g(\mathbf{c})$ and $g(\mathbf{d})$, is ambiguous; $\mathbf{T}_{1}$ and $\mathbf{T}_{2}$ illustrate choices giving rise to backward continuations which are not order isomorphic.

Observe that a backward continuation is not always unambiguous; given a class, if we wish to find a preimage in a $\mathcal{L}$-unlinked class $U$ containing a last class of $\mathcal{L}$, different admissible extensions can give rise to finite laminations that are not order isomorphic. In such a situation, we must specify exactly where the pull back of the image class is located in $U$ with respect to all last classes contained in $U$. See Figure 2.2. 1 .

Definition 2.2.2. For a critical lamination $(\mathcal{L}, g)$ with critical leaves $\mathcal{C}=$ $\{\mathbf{c}, \mathbf{d}\}$, a $\mathcal{L}$-itinerary is a finite string $i_{0} i_{1} \ldots i_{n}$ of $\mathcal{C}$-unlinked classes and critical classes. A $\mathcal{L}$-itinerary which contains no critical classes is called non-critical. A $\mathcal{L}$-itinerary whose last element, but no other, is a critical leaf is called end-critical. We associate to $x \in \mathbb{S}^{1}$ (not necessarily contained in any $\mathcal{L}$-class) the maximal $\mathcal{L}$-itinerary $\operatorname{itin}_{\max }(x)=I_{0} I_{1} I_{2} \ldots$ of $x$, which is the (typically finite) maximal well-defined sequence of $\mathcal{C}$-unlinked classes 
and critical classes such that for every admissible extension $F$ of $g$ we have that $F^{j}(x) \in I_{j}$; $\operatorname{itin}_{\max }(x)$ begins at the moment zero, i.e. at the $\mathcal{C}$-unlinked class or critical class containing $x$. An initial string of the maximal $\mathcal{L}$ itinerary of $x$ is said to be a $\mathcal{L}$-itinerary of $x$.

An arbitrary itinerary is not assumed to be realized by an element of a critical lamination, but is considered only as a potential itinerary of an element of $\mathcal{L}$. However, if $\mathbf{m}$ is a $\mathcal{L}$-class we can also define for it an itinerary of different length. Namely, the true $\mathcal{L}$-itinerary $\operatorname{itin}_{\text {true }}(\mathbf{m})$ is the maximal sequence of $\mathcal{C}$-unlinked classes and critical classes containing the $g$-images of $\mathbf{m}$ as long as these $g$-images are still $\mathcal{L}$-classes. Since the $g$ images of $\mathcal{L}$-classes are $\mathcal{L}$-classes, $\operatorname{itin}_{\text {true }}(\mathbf{m})$ coincides with an appropriate initial string of $\operatorname{itin}_{\max }(x)$ for each $x \in \mathbf{m}$. For example, the true itinerary of a last class has only one entry, while its maximal itinerary may have many entries. If $\mathcal{L}$ is fixed, we may talk about itineraries without explicitly mentioning $\mathcal{L}$.

2.3. Main continuation lemma. Here, we describe the main ingredients used in the construction of finite laminations in the next section. First we define the following concept. Suppose that $A$ and $B$ are either edges or classes of a finite lamination $\mathcal{L}$ such that $A \cap B$ is at most a point. Consider components of $\overline{\mathbb{D}} \backslash(\mathrm{Ch}(A) \cup \mathrm{Ch}(B))$ and choose among them the unique component $X$ which borders both $A$ and $B$. This component $X$ is called the part of $\overline{\mathbb{D}}$ between $A$ and $B$. Also, recall that by leaves we mean all $\mathcal{L}$-classes consisting of two points (as well as their convex hulls) while by edges we mean boundary chords of convex hulls of $\mathcal{L}$-classes.

Definition 2.3.1 (Adjacent, leaf-like). Two classes (or edges) are adjacent if the part of $\overline{\mathbb{D}}$ between them is disjoint from $\mathcal{L}$. A triple $\mathbf{T}$ is leaf-like if exactly one of the components of $\mathbb{S}^{1} \backslash \mathbf{T}$ is disjoint from $\mathcal{L}$; if $\mathbf{T}$ is leaf-like and adjacent to a bud $\mathrm{x}$, then the short edge of $\mathrm{T}$ is the edge $\mathrm{s}$ of $\mathrm{T}$ adjacent to $\mathrm{x}$, the long edge of $\mathrm{T}$ is the edge $\ell$ of $\mathrm{T}$ separating $\mathrm{T} \backslash \boldsymbol{\ell}$ (and $\mathrm{x}$ ) from the rest of $\mathcal{L}$, and the empty edge of $\mathbf{T}$ is the remaining edge of $\mathbf{T}$. A leaf is always considered to be leaf-like. The arc under a leaf $\mathbf{t}$ is the (open) component of $\mathbb{S}^{1} \backslash \mathbf{t}$ which contains no class of $\mathcal{L}$, if such an interval exists. Finally, if $\bar{a}$ is an edge of a triple $\mathbf{T}$, then the arc under $\bar{a}$ is the component of $\mathbb{S}^{1} \backslash \bar{a}$ which does not contain $\mathbf{T} \backslash \bar{a}$. Observe that the notion of the arc under a leaf and that of the arc under an edge of a triangle have somewhat different meaning. These notions are illustrated in Figure 2.3.1.

Definition 2.3.2 (c-lamination). Let $(\mathcal{L}, g)$ be a finite cubic WT-lamination. We say that $(\mathcal{L}, g)$ is a c-lamination if there is a triple $\mathbf{T}^{\mathbf{d}}$, a preimage $\mathbf{m}(\mathcal{L})=\mathbf{m}$ of $\mathbf{c}$, and a preimage $\ell(\mathcal{L})=\ell$ of $\mathbf{d}$ such that the following hold. 


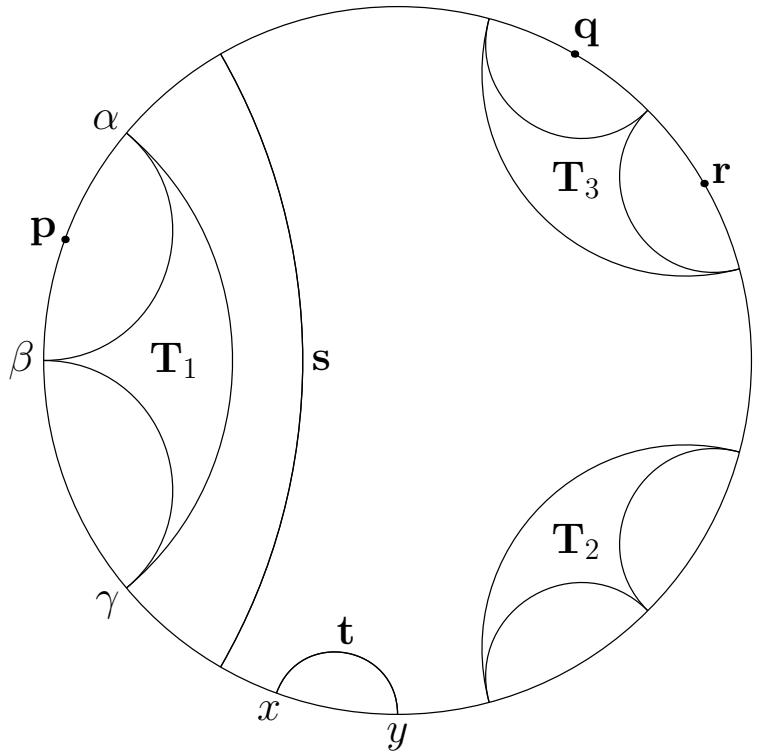

FIGURE 2.3.1. An illustration of concepts from Definition 2.3.1. The finite lamination in question is $\mathcal{L}=$ $\left\{\mathbf{p}, \mathbf{q}, \mathbf{r}, \mathbf{s}, \mathbf{t}, \mathbf{T}_{1}, \mathbf{T}_{2}, \mathbf{T}_{3}\right\}$. The triple $\mathbf{T}_{1}$ is leaf-like, and is adjacent to both the bud $\mathbf{p}$ and the leaf $\mathbf{s}$. Its long edge is therefore $\{\alpha, \gamma\}$, its short edge is $\{\alpha, \beta\}$, and its empty edge is $\{\beta, \gamma\}$. The class $\mathbf{T}_{3}$ is adjacent to both $\mathbf{q}$ and $\mathbf{r}$. The triple $\mathbf{T}_{2}$ is not leaf-like, and is not adjacent to any other class. The arc under the leaf $\mathbf{t}$ is $[x, y]$. There are no arcs under $\mathbf{s}$. The arcs under the different edges of $T_{1}$ are $[\alpha, \beta],[\beta, \gamma]$, and $[\gamma, \alpha]$.

(1) $\mathbf{T}^{\mathbf{d}}$ is adjacent to both $\mathbf{d}^{\prime}$ and $\mathbf{m}$.

(2) If $r>0$ is such that $g^{r}(\mathbf{m})=\mathbf{c}^{\prime}$, then the last triangle $\mathbf{T}^{l}$ is equal to $g^{r}\left(\mathbf{T}^{\mathbf{d}}\right)$. Further, if $0 \leq k \leq r$, then $g^{k}\left(\mathbf{T}^{\mathbf{d}}\right)$ is leaf-like and adjacent to $g^{k}(\mathbf{m})$. In particular, the last triple is leaf-like and adjacent to $\mathbf{c}^{\prime}$.

(3) If $0<k \leq r$, the short edge of $\mathbf{T}^{\mathbf{d}}$ facing $\mathbf{d}^{\prime}$ maps under $g^{k}$ to an empty edge of $g^{k}\left(\mathbf{T}^{\mathbf{d}}\right)$.

(4) The long edge of the last triple is adjacent to $\ell$.

By replacing $\mathbf{c}$ with $\mathbf{d}$ above, we obtain the definition of a d-lamination $\mathcal{L}$. More precisely, we say that $(\mathcal{L}, g)$ is a d-lamination if there is a triple $\mathbf{T}^{\mathbf{c}}$, a preimage $\mathbf{m}(\mathcal{L})=\mathbf{m}$ of $\mathbf{d}$, and a preimage $\ell(\mathcal{L})=\ell$ of $\mathbf{c}$ such that the following holds.

(1) $\mathbf{T}^{\mathbf{c}}$ is adjacent to both $\mathbf{c}^{\prime}$ and $\mathbf{m}$. 


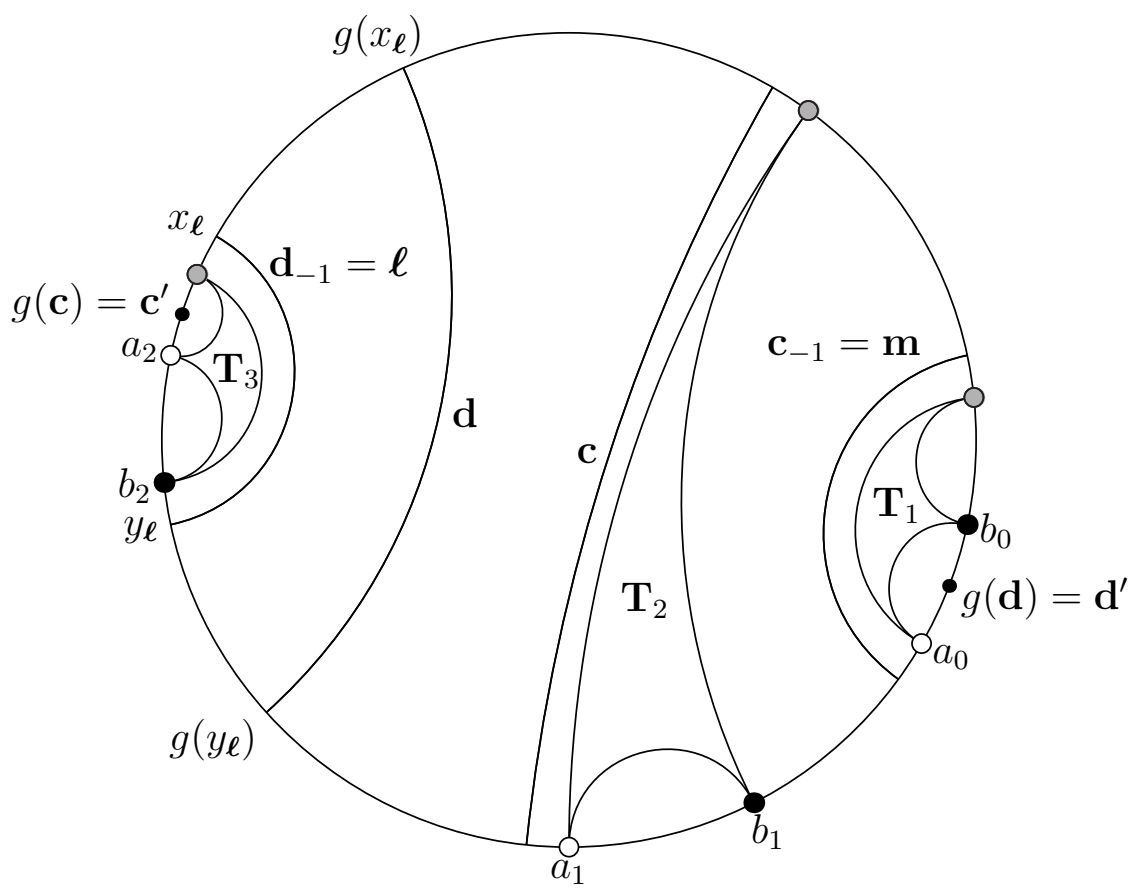

FIGURE 2.3.2. A simple c-lamination. Like-shaded vertices are in the same orbit. The monotone sequence of intervals is $\left(\left[a_{0}, b_{0}\right],\left[a_{1}, b_{1}\right],\left[a_{2}, b_{2}\right],\left[g\left(x_{\ell}\right), g\left(y_{\ell}\right)\right]\right), g^{2}(\mathbf{m})=\mathbf{c}^{\prime}$ and $r=2$. The monotone itinerary is $(C, C, D, D)$, where $C$ denotes the $\{\mathbf{c}, \mathbf{d}\}$-unlinked class whose boundary is $\mathbf{c}$, and likewise $D$ for $\mathbf{d}$.

(2) If $r>0$ is such that $g^{r}(\mathbf{m})=\mathbf{d}^{\prime}$, then the last triangle $\mathbf{T}^{l}$ is equal to $g^{r}\left(\mathbf{T}^{\mathbf{c}}\right)$. Further, if $0 \leq k \leq r$, then $g^{k}\left(\mathbf{T}^{\mathbf{c}}\right)$ is leaf-like and adjacent to $g^{k}(\mathbf{m})$. In particular, the last triple is leaf-like and adjacent to $\mathbf{d}^{\prime}$.

(3) If $0<k \leq r$, the short edge of $\mathbf{T}^{\mathbf{c}}$ facing $\mathbf{c}^{\prime}$ maps under $g^{k}$ to an empty edge of $g^{k}\left(\mathbf{T}^{\mathbf{c}}\right)$.

(4) The long edge of the last triple is adjacent to $\ell$.

Given a c-lamination $(\mathcal{L}, g)$, we observe that a particular sequence of intervals describes the maximal $\mathcal{L}$-itinerary of $\mathbf{d}^{\prime}$ in the sense of Definition 2.2.2. Let $\left\{a_{0}, b_{0}\right\}$ denote the endpoints of the short edge of $\mathbf{T}^{\mathbf{d}}$, so $\mathbf{d}^{\prime} \in K_{0}=\left[a_{0}, b_{0}\right]$. As in Definition 2.3.2, let $r$ denote the integer such that $g^{r}\left(\mathbf{T}^{\mathbf{d}}\right)=\mathbf{T}^{l}$. We then set $a_{k}=g^{k}\left(a_{0}\right)$ and $b_{k}=g^{k}\left(b_{0}\right)$ for each $k \leq r$. Since $\left\{a_{k}, b_{k}\right\}$ is the empty edge of $g^{k}\left(\mathbf{T}^{\mathbf{d}}\right)$, it is apparent from the definition of a c-lamination that $G^{k}\left(\left[a_{0}, b_{0}\right]\right)=\left[a_{k}, b_{k}\right]=K_{k}$ for any admissible extension $G$ of $g$ and any $k \leq r$. 
Let $\left\{x_{\ell}, y_{\ell}\right\}$ be the endpoints of $\ell$ labeled so that $K_{r} \subset\left[x_{\ell}, y_{\ell}\right]$. Then $G^{r}\left(\mathbf{d}^{\prime}\right) \in K_{r} \subset\left[x_{\ell}, y_{\ell}\right]$ for any admissible extension $G$ of $g$. In other words, the first $r$ entries of the maximal $\mathcal{L}$-itinerary of $\mathbf{d}^{\prime}$ are uniquely determined by the images of the short edge of $\mathbf{T}^{\mathbf{d}}$ facing $\mathbf{d}^{\prime}$, i.e., empty edges of triples $g^{i}\left(\mathbf{T}^{\mathbf{d}}\right)$ (see Definition 2.3.2(4)). Then, from the moment $r$ on, the images of $\mathrm{d}^{\prime}$ cannot be described through images of edges of $\mathrm{T}^{l}$ because $\mathrm{T}^{l}$ is the last triple and images of $\mathrm{T}^{l}$ are not defined.

However images of $\ell$ are defined and force the next uniquely determined string of the maximal $\mathcal{L}$-itinerary of $\mathbf{d}^{\prime}$. More precisely, we are guaranteed that $G^{r+k+1}\left(\mathbf{d}^{\prime}\right) \in\left[g^{k+1}\left(x_{\ell}\right), g^{k+1}\left(y_{\ell}\right)\right]$ if the interval $\left[g^{k}\left(x_{\ell}\right), g^{k}\left(y_{\ell}\right)\right]$ contains no critical leaf. We define $K_{r+1}=\left[g\left(x_{\ell}\right), g\left(y_{\ell}\right)\right]$. If $K_{r+k}=$ $\left[g^{k}\left(x_{\ell}\right), g^{k}\left(y_{\ell}\right)\right]$ is defined and contains no critical leaf, then we set $K_{r+k+1}=$ $\left[g^{k+1}\left(x_{\ell}\right), g^{k+1}\left(y_{\ell}\right)\right]$. Since $\ell$ is eventually critical, for some $N$ we have that $K_{N}$ contains a critical leaf, and the process stops. If $G$ is any admissible extension of $g$, then $N$ is maximal such that $\left.G^{N}\right|_{K_{0}}$ is one-to-one. Set $N(\mathcal{L})=N$.

Recall that $\mathcal{C}=\{\mathbf{c}, \mathbf{d}\}$. It follows that if $K_{N}$ is not the closure of a connected $\mathcal{C}$-unlinked class then $i_{0} \ldots i_{N-1}$ is the maximal $\mathcal{L}$-itinerary of $\mathbf{d}^{\prime}$ (and of every point in the interior of $K_{0}$ ). Choose $i_{N}$ to be a connected $\mathcal{C}$-unlinked class contained in $K_{N}$. If $K_{N}$ is the closure of a connected $\mathcal{C}$ unlinked class, then this class must be $i_{N}$ and the maximal $\mathcal{L}$-itinerary of $\mathbf{d}^{\prime}$ is $i_{0} \ldots i_{N-1} i_{N}$.

The above introduced notation (number $r$, intervals $K_{i}=\left[a_{i}, b_{i}\right], \boldsymbol{\ell}=$ $\overline{x_{\ell}, y_{\ell}}$, etc.) will be used throughout the paper when we talk about claminations.

Definition 2.3.3. For a c-lamination $(\mathcal{L}, g)$, call $K_{0}, \ldots, K_{N(\mathcal{L})}$ the monotone sequence of intervals. (As usual, the parenthetically indicated dependence of the intervals or $N$ upon $\mathcal{L}$ may be omitted.) The itinerary $\left.i_{0}^{\text {mon }} \ldots i_{N}^{\text {mon }}\right)=\operatorname{itin}_{\text {mon }}(\mathcal{L})$ is called the monotone itinerary (of $(\mathcal{L}, g)$ ). Define a monotone sequence of intervals and a monotone itinerary of a $\mathbf{d}$ lamination similarly.

Remark 2.3.4. In this remark we use the notation from Definition 2.3.2 (in particular, $r$ below is the positive integer such that $g^{r}(\mathbf{m})=\mathbf{c}^{\prime}$ and $r \leq N)$. We claim that the true itinerary of $\mathbf{m}$ does not have $i_{0}^{\text {mon }} \ldots i_{N}^{\text {mon }}=$ $\operatorname{itin}_{\text {mon }}(\mathcal{L})$ as its initial segment, because the orbit of $\mathbf{m}$ passes through $\mathbf{c}$ in the first $r$ steps, and $\operatorname{itin}_{\text {mon }}(\mathcal{L})$ is non-critical. Let us also show that an initial segment of $\operatorname{itin}_{\text {true }}(\ell)$ cannot coincide with $i_{r}^{\text {mon }} \ldots i_{N}^{\text {mon }}$. This is because either $\overline{i_{N}^{\mathrm{mon}}} \subsetneq K_{N}$, in which case the endpoints of $K_{n}$ (i.e., the endpoints of $g^{N-r}(\ell)$ ) cannot lie within $K_{N}$; or $g^{N-r}(\ell)=\mathbf{d}$, while $\operatorname{itin}_{\text {mon }}(\mathcal{L})$ is not end-critical by definition.

The following is another consequence of the definition. 
Lemma 2.3.5. Let $(\mathcal{L}, g)$ be a c-lamination. Suppose $\left(\mathcal{L}^{\prime}, g^{\prime}\right)$ is a continuation of $(\mathcal{L}, g)$ such that $\mathbf{T}^{l}(\mathcal{L})$ is still adjacent to $\ell(\mathcal{L})$ and to $\mathbf{c}^{\prime}$ in $\mathcal{L}^{\prime}$. If the true $\mathcal{L}^{\prime}$-itinerary of a non-degenerate $\mathcal{L}^{\prime}$-class $\mathbf{z}$ begins with $\operatorname{itin}_{\text {mon }}(\mathcal{L})$, then $\mathrm{z}$ lies in $K_{0}$.

Proof. We will show by induction on $t$ that $\left(g^{\prime}\right)^{N-t}(\mathbf{z}) \subset K_{N-t}$. Since $g^{\prime}$ is a continuation of $g$, we may use $g$ and $g^{\prime}$ interchangeably on $\mathcal{L}$-classes.

That $\left(g^{\prime}\right)^{N}(\mathbf{z}) \subset i_{N}^{\text {mon }} \subset K_{N}$ follows by definition. Suppose by induction that $\left(g^{\prime}\right)^{j+1}(\mathbf{z}) \subset K_{j+1}$ for $0 \leq j<N$. We see that $\left(g^{\prime}\right)^{j}(\mathbf{z})$ is the unique class in $i_{j}^{\text {mon }}$ which maps onto $\left(g^{\prime}\right)^{j+1}(\mathbf{z})$. If $j \neq r$, then $\left(g^{\prime}\right)^{j}(\mathbf{z}) \subset K_{j}$ since $\left.g^{\prime}\right|_{i_{j} \text { mon }}$ is order-preserving. On the other hand, if $j=r$, then $K_{j+1}=\left[g\left(x_{\ell}\right), g\left(y_{\ell}\right)\right]$ and $\left(g^{\prime}\right)^{j}(\mathbf{z}) \subset\left[x_{\ell}, y_{\ell}\right]$ since $\left.g^{\prime}\right|_{i_{r}}$ is order-preserving. However, we assume that $\ell$ is adjacent to $\mathbf{T}^{l}$ and $\mathbf{c}^{\prime}$ in $\mathcal{L}^{\prime}$, and $\left(g^{\prime}\right)^{j}(\mathbf{z})$ is neither $\boldsymbol{\ell}$ nor $\mathbf{c}^{\prime}$ by Remark 2.3.4, so $\left(g^{\prime}\right)^{j}(\mathbf{z}) \subset\left[a_{r}, b_{r}\right]=K_{r}$. Hence, by induction, $\mathbf{z} \subset K_{0}$.

Lemma 2.3.6 is an important inductive step in the construction.

Lemma 2.3.6. Let $(\mathcal{L}, g)$ be a c-lamination, and let $\tau=j_{0} \ldots j_{M} \mathbf{c}$ be an end-critical $\mathcal{L}$-itinerary where $M \leq N(\mathcal{L})-2$. Then there is a backward continuation $\left(\mathcal{L}^{\prime}, g^{\prime}\right)$ containing a leaf $\ell\left(\mathcal{L}^{\prime}\right)$ such that the following claims hold.

(1) The leaf $\ell\left(\mathcal{L}^{\prime}\right)$ is a $g^{\prime}$-preimage of $\mathbf{c}$, and every leaf in $\mathcal{L}^{\prime} \backslash \mathcal{L}$ is a forward image of $\ell\left(\mathcal{L}^{\prime}\right)$.

(2) The leaf $\ell\left(\mathcal{L}^{\prime}\right)$ is adjacent to $\mathbf{d}^{\prime}$ and $\mathrm{T}^{\mathrm{d}}$.

(3) The true itinerary of $\ell\left(\mathcal{L}^{\prime}\right)$ begins with $\left(i_{0}^{\mathrm{mon}}, \ldots, i_{N}^{\mathrm{mon}}\right)$, ends with $\tau$, and these two segments of the itinerary are disjoint.

(4) The common last triple $T^{l}(\mathcal{L})=T^{l}\left(\mathcal{L}^{\prime}\right)$ of both laminations is still adjacent in $\mathcal{L}^{\prime}$ to both $\ell(\mathcal{L})$ and $\mathbf{c}^{\prime}$.

(5) The length of the maximal $\mathcal{L}^{\prime}$-itinerary of $\mathbf{d}^{\prime}$ is of length at least $N(\mathcal{L})+2$.

Proof. We will construct $\left(\mathcal{L}^{\prime}, g^{\prime}\right)$ by adding a sequence of consecutive pullbacks of $\mathrm{c}$ until we come to the leaf $\ell\left(\mathcal{L}^{\prime}\right)$. We will use the following rule when dealing with ambiguous pullbacks: any pullback into $\left(x_{\ell}, y_{\ell}\right)$ must be under the empty edge of $\mathbf{T}^{l}(\mathcal{L})$. (Any pullbacks into $K_{0}$ would also be ambiguous, but such will not occur until the last pullback we take in this proof.)

Take iterative preimages of $\mathbf{c}$ along the itinerary $\tau$, using the rules above for ambiguous pullbacks. Note that no pullback is taken into the interval $K_{0}$, since $\tau$ is too short to contain the itinerary of any point in $K_{0}$. Denote the leaf so obtained by $\mathbf{c}^{\tau}$. (Note that part or all of the orbit of $\mathbf{c}^{\tau}$ may already be represented in $\mathcal{L}$; we do not add extra copies in order to preserve that $\left(\mathcal{L}^{\prime}, g^{\prime}\right)$ is a degree 3 finite lamination.) 
Next we consider the problem of finding a preimage of $\mathbf{c}_{\tau}$ in $K_{0}$ which increases the length of the maximal itinerary of $\mathbf{d}^{\prime}$. First let us show that $i_{0}=\cdots=i_{N}$ is impossible. Recall from the discussion before Definition 2.3.3 that $i_{N}$ is always a connected $\mathcal{C}$-unlinked class contained in $K_{N}$. By the definition of the monotone sequence of intervals, $K_{N-1} \subset i_{N} \subset$ $K_{N}$. Since the map $g$ preserves orientation on $i_{N}$ and $i_{N}$ is an arc, it follows that $K_{0} \subset K_{1} \subset \cdots \subset K_{N-1} \subset K_{N}$. Thus, $K_{1}=g\left(K_{0}\right)$ contains d' and is not the interval under an empty edge of $g\left(\mathbf{T}^{\mathbf{d}}\right)$, a contradiction with the definition of a c-lamination.

Thus, we may assume that some of the sets $i_{0}^{\text {mon }}, \ldots, i_{N}^{\text {mon }}$ are distinct. Choose a $\mathcal{C}$-unlinked class $C \neq i_{N-1}^{\text {mon }}$ which does not contain the common image of the endpoints of $i_{N}^{\mathrm{mon}}$, and choose a $\mathcal{C}$-unlinked class $Z$ which is neither $i_{0}^{\text {mon }}$ nor $i_{N-1}^{\text {mon }}$. Then, if $k>1$ is $\operatorname{such}_{\text {that }} \operatorname{itin}_{\operatorname{mon}}(\mathcal{L})$ contains no string of $Z$ 's of length $k$ (denoted $Z^{k}$ ), we choose the itinerary $\tau^{\prime}=$ $i_{0} \ldots i_{N} C Z^{k} \tau$. Let us show that $\tau^{\prime}$ contains exactly one copy of the string $i_{0}^{\text {mon }} \ldots i_{N-1}^{\text {mon }}$. Indeed, $i_{1}^{\text {mon }} \ldots i_{N}^{\text {mon }} \neq i_{0}^{\text {mon }} \ldots i_{N-1}^{\text {mon }}$ because not all entries $i_{0}^{\text {mon }}, \ldots, i_{N}^{\text {mon }}$ are equal. Also, $i_{2}^{\text {mon }} \ldots i_{N}^{\text {mon }} C \neq i_{0}^{\text {mon }} \ldots i_{N-1}^{\text {mon }}$ because $C \neq$ $i_{N-1}^{\text {mon }}$. No substring in $\tau^{\prime}$ which ends with $Z$ can be a copy of $i_{0}^{\text {mon }} \ldots i_{N-1}^{\text {mon }}$ because $Z \neq i_{N-1}^{\text {mon }}$. No copy of $i_{0}^{\text {mon }} \ldots i_{N-1}^{\text {mon }}$ can contain a string of $k$ $Z$ 's by the choice of $k$. Finally, no copy of $i_{0}^{\text {mon }} \ldots i_{N-1}^{\text {mon }}$ can begin with $Z$, since $Z \neq i_{0}^{\text {mon }}$. So indeed, $\tau^{\prime}$ contains exactly one copy of the string $i_{0}^{\mathrm{mon}} \ldots i_{N-1}^{\mathrm{mon}}$.

Now, construct preimages of $\mathbf{c}_{\tau}$ moving back from $\mathbf{c}_{\tau}$ along the itinerary $i_{0}^{\text {mon }} \ldots i_{N}^{\text {mon }} C Z^{k}$. By the previous paragraph, all such pullbacks are taken outside of $K_{0}$ except the last. The last pullback $\ell\left(\mathcal{L}^{\prime}\right)$ is to be taken in $K_{0}$ by Lemma 2.3.5, and we choose it to be adjacent to $\mathbf{d}^{\prime}$. Call the resulting lamination $\left(\mathcal{L}^{\prime}, g^{\prime}\right)$. Now every point of the component of $\mathbb{S}^{1} \backslash \ell\left(\mathcal{L}^{\prime}\right)$ containing $\mathbf{d}^{\prime}$ has maximal $\mathcal{L}^{\prime}$-itinerary beginning with $i_{0}^{\text {mon }} \ldots i_{N}^{\text {mon }}$ and has length at least $N(\mathcal{L})+2$. This completes the proof.

Given a c-lamination or a d-lamination $\mathcal{L}$, denote by $\overline{\text { lo }}(\mathcal{L})=\overline{\text { lo }}$ the long edge of $\mathbf{T}^{l}=\mathbf{T}^{l}(\mathcal{L})$ and by $\overline{\operatorname{sh}}(\mathcal{L})=\overline{\text { sh }}$ its short edge.

Lemma 2.3.7. Let $(\mathcal{L}, g)$ be a c-lamination, and let $\tau=j_{0} j_{1} \ldots j_{k}$ c be an end-critical $\mathcal{L}$-itinerary for some $k \leq N(\mathcal{L})-2$ (see Definition 2.3.3). Let $t_{0} t_{1} \ldots t_{m} \mathbf{d}$ be an initial segment of the true $\mathcal{L}$-itinerary of $\ell(\mathcal{L})$. Then there is a d-lamination $\left(\mathcal{L}^{\prime \prime}, g^{\prime \prime}\right)$ which continues $(\mathcal{L}, g)$ in two steps: $(1)$ construct $\left(\mathcal{L}^{\prime}, g^{\prime}\right)$ by $\tau$ as in Lemma 2.3.6 and then (2) add, in the appropriate way, a segment of the orbit of $\mathbf{T}^{l}(\mathcal{L})$ which follows the orbit of $\ell(\mathcal{L})$ so that an initial segment of the true $\mathcal{L}^{\prime \prime}$-itinerary of $\mathbf{T}^{l}(\mathcal{L})$ coincides with $t_{0} \ldots t_{m}$. Moreover, the maximal $\mathcal{L}^{\prime \prime}$-itinerary of $\mathbf{d}^{\prime}$ is of length at least $N(\mathcal{L})+2$, and $\ell\left(\mathcal{L}^{\prime \prime}\right)=\ell\left(\mathcal{L}^{\prime}\right)$ has $\tau$ as a subsegment of its true $\mathcal{L}^{\prime \prime}$-itinerary. 
The statement of Lemma 2.3.7 also holds, with appropriate swapping of $\mathbf{c}$ and $\mathbf{d}$ related objects.

Proof. Let $\left(\mathcal{L}^{\prime}, g^{\prime}\right)$ be the backward continuation given by Lemma 2.3.6. We shall continue $\left(\mathcal{L}^{\prime}, g^{\prime}\right)$ to a d-lamination which will be constructed by adding images of $\mathbf{T}^{l}\left(\mathcal{L}^{\prime}\right)$ to $\left(\mathcal{L}^{\prime}, g^{\prime}\right)$ to obtain a d-lamination $\left(\mathcal{L}^{\prime \prime}, g^{\prime \prime}\right)$ with $\ell\left(\mathcal{L}^{\prime}\right)=\ell\left(\mathcal{L}^{\prime \prime}\right)$. Recall that since $\mathcal{L}$ is a c-lamination, there is also a leaf $\ell(\mathcal{L})$ which is different from $\ell\left(\mathcal{L}^{\prime}\right)$. Let $\mathbf{T}^{l}=\mathbf{T}_{n}$.

Let $s$ and $t$ be such that $g^{s}(\boldsymbol{\ell}(\mathcal{L}))=\mathbf{d}$ and $g^{t}(\mathbf{d})=\mathbf{d}^{\prime}$. Since $\overline{\mathrm{lo}}$ is adjacent to $\ell(\mathcal{L})$ and $\overline{\mathrm{sh}}$ is adjacent to the last class $\mathbf{c}^{\prime}$, we can define $\mathbf{T}_{n+1}$, $\ldots, \mathbf{T}_{n+s+t}$ up to isomorphism so that for any $i \in\{0, \ldots, s+t\}$ we have

(1) $\left(g^{\prime \prime}\right)^{i}(\overline{\mathbf{l o}})$ is adjacent to $g^{i}(\boldsymbol{\ell}(\mathcal{L}))$, and

(2) one component of $\mathbb{S}^{1} \backslash\left(g^{\prime \prime}\right)^{i}(\overline{\mathrm{sh}})$ contains no classes of $\mathcal{L}$.

Note that by the properties of c-laminations all leaves $g^{i}(\ell(\mathcal{L}))$ are located outside $\left[x_{\ell}, y_{\ell}\right]$. Hence the construction implies that $\mathbf{T}_{n+i}$ is outside of $\left[x_{\ell}, y_{\ell}\right]$ for all $0<i \leq s+t$, which implies that the short edge $\overline{\mathrm{sh}}$ of $\mathbf{T}_{n}$ is still adjacent to $\mathbf{c}^{\prime}$ in $\mathcal{L}^{\prime \prime}$. It is now easy to verify that $\left(\mathcal{L}^{\prime \prime}, g^{\prime \prime}\right)$ is a d-lamination and has all the desired properties.

Beginning with any c-lamination $\left(\mathcal{L}_{0}, g_{0}\right)$, by Lemma 2.3.7 one can construct an increasing sequence of critical laminations $\left(\left(\mathcal{L}_{i}, g_{i}\right)\right)_{i=1}^{\infty}$, where $\left(\mathcal{L}_{i+1}, g_{i+1}\right)$ continues $\left(\mathcal{L}_{i}, g_{i}\right)$. This is done on the basis of a sequence of end-critical finite itineraries used in the applications of Lemma 2.3.7 as a sequence $\tau$. As we rely on Lemma 2.3.7, we continue a c-lamination to a d-lamination, then to a c-lamination, etc. Each critical lamination contains a triple $\mathbf{T}_{1}$, and the orbit of $\mathbf{T}_{1}$ is continued in each successive critical lamination by adding a forward segment to the orbit of $\mathbf{T}^{l}(\mathcal{L})$ which follows the orbit of $\ell(\mathcal{L})$ and has the appropriate initial segment of its true itinerary.

In other words, on each step, when we continue $\mathcal{L}$, we fulfill two tasks: (1) create the leaf $\ell\left(\mathcal{L}^{\prime}\right)=\ell\left(\mathcal{L}^{\prime \prime}\right)$ which has any given subsegment in its true itinerary, and (2) add a segment of the orbit of $\mathbf{T}^{l}(\mathcal{L})$ which has a subsegment coinciding with the appropriate (up to the critical leaf) subsegment of the true itinerary of $\ell(\mathcal{L})$. To go on with this construction we need to choose finite itineraries which determine the construction on each step. We can choose them to cover all possible itineraries. Then applying the construction infinitely many times we obtain the limit lamination $\bigcup_{i=1}^{\infty} \mathcal{L}_{i}$ equipped with the limit map $g$, containing (combinatorially) a wandering triangle which has an itinerary in which any finite itinerary shows at least once.

\section{REALIZATION AND DENSITY}


3.1. Realizing the combinatorial lamination. In this section, we show that any c-lamination $\left(\mathcal{L}_{0}, g_{0}\right)$ can be continued (up to conjugacy by an order isomorphism) to a $\sigma_{3}$-invariant lamination $\mathcal{L}$ containing a wandering triangle whose forward orbit is dense in the lamination. We first construct a combinatorial lamination $\left(\mathcal{L}_{\infty}, g_{\infty}\right)$ with appropriate properties by repeated application of Lemma 2.3.7. We show that $g_{\infty}$ restricted to the forward orbit of $\mathbf{T}_{1}$ satisfies an expansion property (see Definition 3.1.2), and is therefore conjugate to a restriction of $\sigma_{3}$ to some subset $A$. The 3 -invariant lamination $\mathcal{L}$ will be the closure of the induced lamination on $A$.

Recall that end-critical itineraries were defined in Definition 2.2.2.

Definition 3.1.1. A sequence $\left(\tau_{n}\right)_{n=0}^{\infty}$ of end-critical itineraries ending in c is called full if the length of $\tau_{n}$ converges to infinity and for any finite precritical itinerary $\tau$ ending in $\mathbf{c}$ there is an integer $n$ such that the itinerary $\tau_{n}$ ends in $\tau$. Similarly we define a full sequence of pairwise distinct precritical itineraries ending in $\mathbf{d}$.

Let $\left(\mathcal{L}_{0}, g_{0}\right)$ be fixed for the rest of the section; we assume that the lengths of the itineraries of $\mathbf{c}$ and $\mathbf{d}$ are both greater than two. Choose a full sequence of itineraries $\left(\tau_{n}^{\mathbf{c}}\right)_{n=0}^{\infty}$ ending in $\mathbf{c}$ such that the following hold.

- The length of $\tau_{0}^{\mathbf{c}}$ is less than the length of the $\mathcal{L}_{0}$-itinerary of $\mathbf{d}^{\prime}$ by at least 2 , and

- The length of $\tau_{i+1}^{\mathbf{c}}$ is at most one more than the length of $\tau_{i}^{\mathbf{c}}$.

Similarly define $\left(\tau_{n}^{\mathbf{d}}\right)_{n=1}^{\infty}$. We then inductively define laminations $\left(\left(\mathcal{L}_{i}, g_{i}\right)\right)_{i=1}^{\infty}$, where even indices correspond to c-laminations and odd indices correspond to d-laminations, as follows.

- If $\mathcal{L}_{i}$ is defined for an even integer $i=2 k$, we use Lemma 2.3.7 with $\tau_{k}^{\mathbf{c}}$ to obtain a d-lamination $\left(\mathcal{L}_{i+1}, g_{i+1}\right)$.

- If $\mathcal{L}_{i}$ is defined for an odd integer $i=2 k-1$, we use Lemma 2.3.7 with $\tau_{k}^{\mathrm{d}}$ to obtain a c-lamination $\left(\mathcal{L}_{i+1}, g_{i+1}\right)$.

Then $\bigcup \mathcal{L}_{i}=\mathcal{L}_{\infty}$ with the natural map $g_{\infty}$ is an infinite combinatorial lamination with a wandering class $\mathbf{T}_{1}$ and a full set of preimages of both $\mathbf{c}$ and $d$, i.e., exactly one leaf corresponding to each end-critical $\mathcal{L}_{0}$-itinerary.

Due to the properties of $\mathbf{c}$ - and d-laminations, the orbit of $\mathbf{T}_{1}$ in $\left(\mathcal{L}_{\infty}, g_{\infty}\right)$ is most easily analyzed in terms of "closest approaches". For example, $\mathbf{T}^{l}\left(\mathcal{L}_{2 k}\right)$ is adjacent to $\mathbf{c}^{\prime}$ in $\mathcal{L}_{2 k}$, so this constitutes a closest approach in the lamination $\mathcal{L}_{2 k}$. These sequences of closest approaches are particularly important to us, so we will keep two sequences in mind: let $\left(p_{n}\right)_{n=0}^{\infty}$ be the sequence so that $\mathbf{T}_{p_{n}}=\mathbf{T}^{l}\left(\mathcal{L}_{n}\right)$, and set $\left(k_{n}\right)_{n=0}^{\infty}$ to be the sequence so that $\mathbf{T}_{k_{n}}$ is adjacent in $\mathcal{L}_{n}$ to the appropriate critical leaf, following its orbit in a leaf-like manner to $\mathbf{c}^{\prime}$ or $\mathbf{d}^{\prime}$. Hence, we have $k_{1}<p_{1}<k_{2}<p_{2}<\ldots$. Further $p_{1}-k_{1}=p_{3}-k_{3}=\cdots=p_{2 n+1}-k_{2 n+1}=\cdots$, and $p_{2}-k_{2}=$ 
$p_{4}-k_{4}=\ldots=p_{2 n}-k_{2 n}=\cdots$ since these are the lengths of the segments of orbits from $\mathbf{c}$ to $\mathbf{c}^{\prime}$, and $\mathbf{d}$ to $\mathbf{d}^{\prime}$, respectively.

Recall that an edge (termed the short edge) of $\mathbf{T}_{k_{n}+1}$ is adjacent in $\mathcal{L}_{n}$ to the image of a critical leaf, so no previous triple lies in the interval underneath that edge. In continuing its orbit, the images of this edge are adjacent to the images of the critical leaf, so no previous triple lies under the corresponding edges of $\mathbf{T}_{k_{n}+2}, \mathbf{T}_{k_{n}+3}, \ldots, \mathbf{T}_{p_{n}}$. Similarly, the part of the disk between $\mathbf{T}_{p_{n}}$ and $\ell\left(\mathcal{L}_{n}\right)$, as well as the part of the disk between their first $p_{n+1}-p_{n}$ images, contains no previous triple. Observe that $p_{n+1}-p_{n} \rightarrow \infty$, since $\left(p_{2 n}\right)_{n=1}^{\infty}$ and $\left(p_{2 n+1}\right)_{n=1}^{\infty}$ are monotone and must eventually accommodate all itineraries $\tau_{i}^{\mathbf{c}}$ and $\tau_{i}^{\mathbf{d}}$.

We will work with the lamination $\mathcal{L}_{\mathbf{T}}=\left\{\mathbf{T}_{n} \mid n \geq 1\right\}$ with basis $A_{\mathbf{T}}=\bigcup_{n=1}^{\infty} \mathbf{T}_{n}$ and the map $g_{\mathbf{T}}=\left.g_{\infty}\right|_{A}$. Note that $A_{\mathbf{T}}$ is forward-invariant under the map $g_{\mathbf{T}}$. We wish to show that $g_{\mathbf{T}}$ is conjugate to a restriction of $\sigma_{3}$. To proceed we need Theorem 3.1.4, to state it we need Definitions 3.1.2 and 3.1.3. Recall that maps of degree 3 are defined right before Definition 2.1.4. Below we work with a set $A \subset \mathbb{S}^{1}$ which is considered with the circular order on it; the topology of $A$ as a subspace of $\mathbb{S}^{1}$ is ignored.

Definition 3.1.2. A map $f: A \rightarrow A$ of degree 3 is said to be topologically exact if for each pair of distinct points $x, y \in A$ there exists an integer $n \geq 1$ such that either $f^{n}(x)=f^{n}(y)$, or $f\left(\left[f^{n}(x), f^{n}(y)\right]_{A}\right) \not \subset$ $\left[f^{n+1}(x), f^{n+1}(y)\right]_{A}$. If we want to emphasize the precise value of $n$, we say that $f$ maps $[x, y]_{A}$ out of order in $n+1$ steps.

Definition 3.1.3 ( $\sigma$-extendable). A degree 3 map $f: A \rightarrow A, A \subset \mathbb{S}^{1}$ is $\sigma$ extendable if for some $\sigma_{3}$-invariant set $A^{\prime} \subset \mathbb{S}^{1}$ the map $\left.f\right|_{A}$ is conjugate to the map $\left.\sigma_{3}\right|_{A^{\prime}}: A^{\prime} \rightarrow A^{\prime}$ and the conjugation is a circular order preserving bijection. Any such $A^{\prime}$ is called an embedding of $A$ into $\sigma_{3}$.

We can now state an important technical result proven in [4].

Theorem 3.1.4 ([4, Theorem 3.3]). If $f: A \rightarrow A \subset \mathbb{S}^{1}$ is a topologically exact map of degree 3 , and $A$ is countable without fixed points, then $f$ is $\sigma$-extendable.

Lemma 3.1.5. The map $g_{\mathbf{T}}$ is $\sigma$-extendable of degree 3 .

Proof. Let $x, y \in A_{\mathrm{T}}$ be distinct points. We consider two main cases: either $[x, y]_{A_{\mathbf{T}}}$ contains the edge of a triple or $[x, y]_{A_{\mathbf{T}}}$ contains vertices from two distinct triples.

First, suppose that $[x, y]_{A_{\mathbf{T}}}$ contains the edge $\{a, b\}$ of a triple $\mathbf{T}_{k} \in \mathcal{L}_{i}$. The idea of the proof in this case is based upon the fact that, by construction, every edge of a triangle eventually becomes a long edge, at which point the interval underneath which will map out of order. To make this more precise, 
assume that $\mathbf{T}_{k} \in \mathcal{L}_{i}$ where $i$ is minimal and $[a, b] \subset[x, y]$. Then $\mathbf{T}_{k}$ maps to $\mathbf{T}_{n}=\mathbf{T}^{l}\left(\mathcal{L}_{i}\right)$; suppose that $[x, y]_{A_{\mathbf{T}}}$ has not mapped out of order by this time. Let $a^{\prime}=\left(g^{\prime}\right)^{n-k}(a)$ and $b^{\prime}=\left(g^{\prime}\right)^{n-k}(b)$. If $\left[a^{\prime}, b^{\prime}\right]_{A_{\mathbf{T}}}$ contains a critical leaf, then $[x, y]_{A_{\mathbf{T}}}$ maps out of order in $n-k+1$ steps. Otherwise, we have three cases.

(1) Suppose $\left\{a^{\prime}, b^{\prime}\right\}$ is the empty edge of $\mathbf{T}_{n}$, i.e., $\left[a^{\prime}, b^{\prime}\right]$ contains no classes of $\mathcal{L}_{i}$, and the other point of $\mathbf{T}_{n}$ is not in $\left[a^{\prime}, b^{\prime}\right]$. Considering $\mathbf{T}_{n}$ in $\mathcal{L}_{i+1}$, we see by Lemma 2.3.7 that $\left\{a^{\prime}, b^{\prime}\right\}$ maps to the long edge $\left\{a^{\prime \prime}, b^{\prime \prime}\right\}$ of $\mathbf{T}^{l}\left(\mathcal{L}_{i+1}\right)$. Since order is preserved, $\left[a^{\prime \prime}, b^{\prime \prime}\right]$ does not contain the other point of $\mathbf{T}^{l}\left(\mathcal{L}_{i+1}\right)$, so $\left[a^{\prime \prime}, b^{\prime \prime}\right]$ contains both critical leaves. Since $\left[a^{\prime \prime}, b^{\prime \prime}\right]_{A_{\mathbf{T}}}$ maps out of order in one step, $[x, y]_{A_{\mathbf{T}}}$ eventually maps out of order.

(2) Suppose $\left\{a^{\prime}, b^{\prime}\right\}$ is the short edge of $\mathbf{T}_{n}$. Then, by Lemma 2.3 .7 $\left\{a^{\prime}, b^{\prime}\right\}$ maps to the empty edge of $\mathbf{T}^{l}\left(\mathcal{L}_{i+1}\right)$. By the previous case, we see that $[x, y]_{A_{\mathbf{T}}}$ eventually maps out of order.

(3) Suppose that $\left\{a^{\prime}, b^{\prime}\right\}$ is the long edge of $\mathbf{T}_{n}$. Since $\left[a^{\prime}, b^{\prime}\right]$ does not contain the endpoints of a critical leaf, $\left[a^{\prime}, b^{\prime}\right]$ must contain the empty edge of $\mathbf{T}_{n}$. We have therefore already shown that $\left[a^{\prime}, b^{\prime}\right]_{A}$, and therefore $[x, y]_{A_{\mathbf{T}}}$, eventually maps out of order.

Suppose now that $x$ and $y$ lie in different triangles $\mathbf{T}_{m_{1}}$ and $\mathbf{T}_{m_{2}}$ with $m_{1}<m_{2}$. In this case the idea of the proof is based upon the fact that the arc $[x, y]$ will eventually cover an endpoint of a critical leaf, then its last critical value which, by construction, implies that it will cover an edge of a triangle and by the previous case will map out of order. The formal argument follows. Assume by way of contradiction that $[x, y]_{A_{\mathbf{T}}}$ never maps out of order. Let $\mathcal{L}_{i}$ be the first lamination in which $\mathbf{T}_{m_{2}}$ appears. Then $\mathbf{T}_{m_{2}}$ eventually maps to the last triple $\mathbf{T}_{m_{2}+k}$ of $\mathcal{L}_{i}$ which is leaf-like adjacent to a last image of a critical leaf (say $\mathbf{c}^{\prime}$ ). Then, since $\mathcal{L}_{i}$ is a c-lamination, there is a, say, $n$-th pullback of $\mathbf{d}$ which is leaf-like adjacent to the long (in the sense of $\mathcal{L}_{i}$ ) edge of $\mathbf{T}_{m_{2}+k}$. This pullback separates $\mathbf{T}_{m_{2}+k}$ from all triples in $\mathcal{L}_{i}$, including $\mathbf{T}_{m_{1}+k}$, so the interval $\left[g^{k}(x), g^{k}(y)\right]$ contains one of its endpoints. By the assumption we get that $\left[g^{k+n+1}(x), g^{k+n+1}(y)\right]$ contains the critical value $g(\mathbf{c})$ in its interior. By construction, we see that $\left[g^{k+n+1}(x), g^{k+n+1}(y)\right]$ contains infinitely many triangles and hence by the previous paragraph a higher power of $g$ will map $[x, y]_{A_{\mathbf{T}}}$ out of order.

Since each interval between points of $A_{\mathrm{T}}$ eventually maps out of order, $g$ is topologically exact. This proves that $g$ is $\sigma$-extendable by Theorem 3.1.4.

Hence, $g_{\mathbf{T}}$ is conjugate to a restriction $\left.\sigma_{3}\right|_{A}$ via circular order isomorphism $h: A_{\mathbf{T}} \rightarrow A$ for some $A \subset \mathbb{S}^{1}$. The map $h$ is not unique; from now 
on we fix it. Set $\hat{\mathbf{T}}_{1}=h\left(\mathbf{T}_{1}\right)$. Let us study properties of the $\sigma_{3}$-orbit of $\hat{\mathbf{T}}_{1}$ and its limit leaves.

Lemma 3.1.6. There are disjoint and unlinked critical chords $\hat{\mathbf{c}}$ and $\hat{\mathbf{d}}$ such that

$$
\sigma_{3}^{k_{2 n}}\left(\hat{\mathbf{T}}_{1}\right) \rightarrow \hat{\mathbf{c}} \text { and } \sigma_{3}^{k_{2 n+1}}\left(\hat{\mathbf{T}}_{1}\right) \rightarrow \hat{\mathbf{d}}
$$

Proof. We will first find $\hat{\mathbf{c}}$. Let $\left(k_{n_{i}}\right)_{i=1}^{\infty}$ denote a subsequence such that $\left\{\hat{\mathbf{T}}_{k_{n_{i}}} \mid i \geq 0\right\}$ are on the same side of $\mathbf{c}$ in $\mathcal{L}_{\infty}$. Since $h$ preserves order on $A_{\mathbf{T}}$, it follows that $\sigma_{3}^{k_{n_{i}}}\left(\hat{\mathbf{T}}_{1}\right)$ converges to a chord $\hat{\mathbf{c}}$. Indeed, since $\sigma_{3}^{m}\left(\hat{\mathbf{T}}_{1}\right)$ are pairwise disjoint convex sets, a subsequence of such sets can only converge to a chord or a point in the circle. However, by construction on either side of $\mathbf{c}$ in the combinatorial model there are images of $\mathbf{T}_{1}$. Hence the limit of $\sigma_{3}^{k_{n}}\left(\hat{\mathbf{T}}_{1}\right)$ must be a chord $\hat{\mathbf{c}}$. In order to see that $\hat{\mathbf{c}}$ is critical we will use that the edges of triangles under which $\sigma_{3}(\hat{\mathbf{c}})$ is located require more and more time to cover $\mathbf{T}_{1}$ and, hence, $\sigma_{3}(\hat{\mathbf{c}})$ must be a point as desired. Now we will implement this idea.

Suppose for contradiction that $\hat{\mathbf{c}}$ is not critical. Then a non-degenerate interval $I$ lies under $\sigma_{3}(\hat{\mathbf{c}})$, so there is a minimal $N>0$ such that $\sigma_{3}^{N}(I)$ contains $\mathbf{T}_{1}$. This implies that one of the first $N$ images of the short edge of $\mathbf{T}_{k_{n_{i}}}$ is neither short nor empty in $\mathcal{L}_{n_{i}+1}$, with the interval underneath either mapping out of order or containing $\mathbf{T}_{1}$. This contradicts the details of the construction; recall that $p_{n_{i}}-k_{n_{i}+1} \rightarrow \infty$ so we can choose $i$ so that $p_{n_{i}}-k_{n_{i}+1}>N$. Then the short edge of $\mathbf{T}_{k_{n_{i}}}$ is short on the segment of its orbit from $k_{n_{i}}+1$ to $p_{n_{i}}$ in $\mathcal{L}_{n_{i}}$ and is empty on the segment from $p_{n_{i}}+1$ to $k_{n_{i}+1}$ in the lamination $\mathcal{L}_{n_{i}+1}$, and therefore does not contain $\mathbf{T}_{1}$. Therefore, $\hat{\mathbf{c}}$ is a critical leaf.

Let $\hat{\mathbf{d}}$ be a critical chord obtained similarly from $\mathbf{T}_{k_{2 n+1}}$. In principle, it is possible that four chords leaves can arise this way: two for each side of $\mathbf{c}$ and $\mathbf{d}$. However, since $g_{\infty}(\mathbf{c}) \neq g_{\infty}(\mathbf{d})$ and $h$ preserves order, it follows that $\sigma_{3}(\hat{\mathbf{c}}) \neq \sigma_{3}(\hat{\mathbf{d}})$. There is no room for another critical chord unlinked with $\hat{\mathbf{c}}$ and $\hat{\mathbf{d}}$, so $\mathbf{T}_{k_{2 n}} \rightarrow \hat{\mathbf{c}}$ and $\mathbf{T}_{k_{2 n+1}} \rightarrow \hat{\mathbf{d}}$.

Let $\hat{\Theta}$ denote the critical portrait $\{\hat{\mathbf{c}}, \hat{\mathbf{d}}\}$.

Lemma 3.1.7. The critical portrait $\hat{\Theta}$ has aperiodic kneading.

Proof. By Lemma 2.3.7 for each $n$ the first segment of length $p_{2 n+1}-p_{2 n}$ of the itinerary of $\sigma_{3}(\hat{\mathbf{c}})$ equals the itinerary of $\mathbf{T}_{1}$ from $\mathbf{T}_{p_{2 n}}$ to $\mathbf{T}_{p_{2 n+1}}$ as given by $\mathcal{L}_{2 n+1}$. By construction, as we vary $n$, the initial segments of the itinerary of $\sigma_{3}(\hat{\mathbf{c}})$ will have to contain all itineraries $\tau_{i}^{\mathbf{c}}$, so the itinerary of $\hat{\mathbf{c}}$ cannot be periodic. 
According to Theorem 1.2 .5 , there is a unique $\hat{\Theta}$-compatible lamination $\sim_{\hat{\Theta}}=\sim$. The quotient $J_{\sim}=\mathbb{S}^{1} / \sim$ is a dendrite, with corresponding quotient map $p: \mathbb{S}^{1} \rightarrow J_{\sim}$. Denote by $\mathcal{L}_{\sim}$ the collection of (maybe degenerate) boundary chords of convex hulls of all $\sim$-classes. As in [17] the set $\mathcal{L}_{\sim}$ can be interpreted geometrically. For each $\hat{\Theta}$-itinerary $\tau$ there are unique preimages of $\hat{\mathbf{c}}$ and $\hat{\mathbf{d}}$ which are pullbacks corresponding to $\tau$. Note that the closures of the preimages of $\hat{\mathbf{c}}$ and $\hat{\mathbf{d}}$ generate a $\hat{\Theta}$-compatible lamination, which equals $\mathcal{L}_{\sim}$ by the uniqueness of the $\hat{\Theta}$-compatible lamination (Theorem 1.2.5); it is therefore not difficult to see that preimages of $\hat{\mathbf{c}}$ and $\hat{\mathbf{d}}$ are dense in $\mathcal{L}_{\sim}$.

Lemma 3.1.8. The triangle $\hat{\mathbf{T}}_{1}$ is a -class which is a wandering triangle, $\hat{\mathbf{c}}$ and $\hat{\mathbf{d}}$ are the critical $\sim$-classes, and the forward orbit of $p\left(\hat{\mathbf{T}}_{1}\right)$ is condense in $J_{\sim}$.

Proof. By Theorem 1.2.5, $\hat{\mathbf{T}}_{1}$ is contained in a finite $\sim$-class w. Since $\hat{\mathbf{T}}_{1}$ is wandering, so is w. By [3, Theorem A] (see also [8]), it follows that $\hat{\mathbf{T}}_{1}=\mathbf{w}$ and the critical $\sim$-classes are $\hat{\mathbf{c}}$ and $\hat{\mathbf{d}}$.

That the forward orbit of $p\left(\hat{\mathbf{T}}_{1}\right)$ is condense in $J_{\sim}$ is equivalent to the forward orbit of $\hat{\mathbf{T}}_{1}$ being dense in $\mathcal{L}_{\sim}$ in the sense that every leaf in $\mathcal{L}$ can be approximated arbitrary well by an edge of some $\hat{\mathbf{T}}_{N}$. As discussed above, it suffices to show that every critical pullback is approximated by the orbit of the triangle $\hat{\mathbf{T}}_{1}$ arbitrarily well. Choose a precritical itinerary $\tau$ of length $N$ with the last entry $\hat{\mathbf{c}}$ and let $\hat{\ell}_{\tau}$ be the pullback leaf of $\hat{\mathbf{c}}$ in $\mathcal{L}_{\sim}$ with itinerary $\tau$. Since $\hat{\mathbf{c}}$ is a $\sim$-class, so is $\hat{\ell}_{\tau}$.

By construction, for every pullback $\tilde{\mathbf{c}}$ of $\mathbf{c}$ in the combinatorial lamination there is a triangle "close" to it which follows the itinerary of $\tilde{\mathbf{c}}$ up to the point that $\tilde{\mathbf{c}}$ maps to c. This will imply that in the $\sigma_{3}$-implementation of this combinatorial lamination the corresponding triangle can be chosen arbitrary close to the corresponding critical pullback of $\hat{\mathbf{c}}$. The formal proof of this fact is given below.

Choose a sequence of precritical itineraries $\tau_{u_{i}}^{\mathbf{c}}$, whose lengths approach infinity, all of which in the end have a segment coinciding with $\tau$. By construction and Lemma 2.3.7, in the lamination $\mathcal{L}_{2 u_{i}}$ there is a leaf $\ell\left(\mathcal{L}_{2 u_{i}-1}\right)$ which is a pullback of $\mathbf{c}$ exhibiting itinerary $\tau_{u_{i}}^{\mathbf{c}}$ right before it maps to c. This implies that the appropriate image of $\ell\left(\mathcal{L}_{2 u_{i}-1}\right)$ is the leaf $\ell_{\tau}$ with itinerary $\tau$. Moreover, the last triangle $\mathbf{T}^{l}\left(\mathcal{L}_{2 u_{i}-1}\right)$ is adjacent to $\ell\left(\mathcal{L}_{2 u_{i}-1}\right)$ in $\mathcal{L}_{2 u_{i}-1}$, maps to the triangle, denoted here $\mathbf{T}^{i}$, which is adjacent to $\ell^{\tau}$ and then to the triangle $\mathbf{T}_{k_{2 u_{i}}}$ adjacent to $\mathbf{c}$ in $\mathcal{L}_{2 u_{i}}$.

Let us show that then $\hat{\mathbf{T}}^{i}$ converges to $\hat{\ell}_{\tau}$. Indeed, as in the proof of Lemma 3.1.6 we may assume that a sequence of triangles $\hat{\mathbf{T}}^{i}$ converges to 
some chord $\hat{\ell}$ from one side. Then $\sigma_{3}^{N}\left(\hat{\mathbf{T}}^{i}\right)=\hat{\mathbf{T}}_{k_{2 u_{i}}}$ and by continuity $\sigma_{3}^{N}(\hat{\ell})$ equals the limit of the triangles $\hat{\mathbf{T}}_{k_{2 u_{i}}}$ which, by Lemma 3.1.6, is $\hat{\mathbf{c}}$. On the other hand, by construction the itinerary of $\hat{\ell}$ before that moment coincides with $\tau$ up to its last entry. Hence $\hat{\ell}=\hat{\ell}_{\tau}$ as desired. Since $\tau$ was an arbitrary precritical itinerary ending with $\mathbf{c}$ and since the same arguments can be used if it ends with $\mathbf{d}$, we conclude that in fact any pullback leaf of a critical leaf in $\mathcal{L}_{\sim}$ is a limit leaf of the forward orbit of $\hat{\mathbf{T}}_{1}$, and so, as explained above, the forward orbit of $p\left(\hat{\mathbf{T}}_{1}\right)$ is condense in $J_{\sim}$.

Lemma 3.1.9. $\mathcal{L}$ contains an order isomorphic copy of $\left(\mathcal{L}_{0}, g_{0}\right)$.

Proof. The preimage of the sequence $\left(\mathbf{T}_{k_{2 n}}\right)$ corresponding to the itinerary $\tau$ converges to a leaf $\hat{\mathbf{c}}^{\tau}$, and likewise for $\left(\mathbf{T}_{k_{2 n+1}}\right)$ and $\mathbf{d}$. The collection of all such leaves for every itinerary $\tau$ represented in $\left(\mathcal{L}_{0}, g_{0}\right)$, as well as some forward images of $\hat{\mathbf{c}}$ and $\hat{\mathbf{d}}$, then forms an order isomorphic copy of $\left(\mathcal{L}_{0}, g_{0}\right)$ in $\mathcal{L}$. The straightforward details are left to the reader.

Given a full sequence of end-critical itineraries ending in $\mathbf{c}$ and a full sequence of end-critical itineraries ending in $\mathbf{d}$, we can find the critical portrait $\Theta$ from Theorem 3.1 .10 so that a sublamination of $\sim_{\Theta}$ is order isomorphic to the lamination $\left(\mathcal{L}_{\infty}, g_{\infty}\right)$ constructed on the basis of these two full sequences of end-critical itineraries by repeated application of Lemma 2.3.7. Hence, we have proven the following theorem (recall the the notion of condensity was introduced in Subsection 1.2.2.

Theorem 3.1.10. If $\left(\mathcal{L}_{0}, g_{0}\right)$ is a c-lamination, then there exists a critical portrait $\hat{\Theta}$ such that the following hold.

(1) $\hat{\Theta}$ has aperiodic kneading.

(2) $\sim_{\Theta}$ has a wandering class $\mathbf{T}_{1}$ consisting of three points.

(3) The orbit of $\mathbf{T}_{1}$ is condense in the quotient space $\mathbb{S}^{1} / \sim_{\Theta}$.

(4) A subcollection of $\sim$-classes forms a finite dynamical sublamination which is conjugate to $\left(\mathcal{L}_{0}, g_{0}\right)$.

3.2. Locating combinatorial laminations in $\mathcal{A}_{3}$. In this section, we show that the collection of critical portraits corresponding to the $\sigma_{3}$-invariant laminations given by Theorem 3.1 .10 is dense in the space of all critical portraits with the compact-unlinked topology (see Definition 1.2.3). To do so, we take a critical portrait $\Theta$ and construct a c-lamination $(\mathcal{L}, g)$ such that the critical portrait of any $\sigma_{3}$-invariant lamination containing an order isomorphic copy of $(\mathcal{L}, g)$ is close to $\Theta$. The critical portrait of any $\sigma_{3}$-invariant lamination given by Theorem 3.1 .10 for $(\mathcal{L}, g)$ is then close to $\Theta$.

To do so, we make the observation that certain dynamical behaviors in invariant laminations tell us where particular points of the circle can be 
located. For example, if there are points $a, b \in \mathbb{S}^{1}$ such that $\sigma_{3}(a)<a<$ $b<\sigma_{3}(b)$ in counter-clockwise order on the interval $\left[\sigma_{3}(a), \sigma_{3}(b)\right]$, we have either $0 \in(a, b)$ or $1 / 2 \in(a, b)$. We can use this sort of information to pinpoint the locations of critical portraits to high precision. Let $A_{\mathbf{c}}(\Theta)$ be the component of $\mathbb{S}^{1} \backslash \Theta$ which is an arc whose endpoints are the endpoints of c. Define $A_{\mathbf{d}}(\Theta)$ similarly. Given a leaf $\ell$ contained in a connected component $H$ of a $\Theta$-unlinked class, let $I_{\ell}$ be the arc in $H$ with the same endpoints. We often refer to $I_{\ell}$ as being under $\ell$; note that this is compatible with the previous definition of "under a leaf", but not "under the edge of a triple".

Definition 3.2.1 (Settled lamination). Let $(\mathcal{L}, g)$ be a critical lamination with two disjoint critical leaves $\Theta=\{\mathbf{c}, \mathbf{d}\}$ such that the following conditions hold.

(1) At least one of the following conditions hold.

(a) There exists a leaf $\ell_{x} \in \mathcal{L}$ such that $I_{\ell_{x}} \subset I_{g\left(\ell_{x}\right)} \subset A_{\mathbf{c}}(\Theta)$. (This models the case where $I_{\ell_{x}}$ is a short interval containing 0 or $1 / 2$.)

(b) There exists a leaf $\ell_{x} \in \mathcal{L}$ such that $I_{\ell_{x}} \subset I_{g^{2}\left(\ell_{x}\right)} \subset A_{\mathbf{c}}(\Theta)$ and $I_{g\left(\ell_{x}\right)} \subset A_{\mathbf{d}}(\Theta)$. (This models the case where $I_{\ell_{x}}$ is a short interval containing $1 / 4$ or $3 / 4$.)

(2) $(\mathcal{L}, g)$ contains disjoint preimages $\ell_{y}$ and $\ell_{z}$ of $\ell_{x}$, each contained entirely within a connected component of a $\Theta$-unlinked class not coinciding with the $\Theta$-unlinked class which contains $\ell_{x}$.

(3) The finite orbits of $\mathbf{c}$ and $\mathbf{d}$ in $\mathcal{L}$ are disjoint from $I_{\ell_{x}} \cup I_{\ell_{y}} \cup I_{\ell_{z}}$ and do not contain periodic points.

(4) Every class of $\mathcal{L}$ is either an image or a preimage of $\mathbf{c}$ or $\mathbf{d}$.

Then we call $(\mathcal{L}, g)$ a finite settled lamination.

The advantage of settled laminations is that for them one can define not only $\mathcal{L}$-itineraries but also itineraries with respect to the hypothetical points $x, y$, and $z$ represented by $I_{x}, I_{y}$, and $I_{z}$. This can be used to locate, with any given precision, a cubic critical portrait whose geometric lamination contains an order isomorphic copy of $(\mathcal{L}, g)$. Denote by $R_{1 / 2}: \mathbb{S}^{1} \rightarrow \mathbb{S}^{1}$ the rotation $R_{1 / 2}(x)=x+1 / 2 \bmod 1$.

Lemma 3.2.2. Let $\Theta^{\prime}$ be a cubic critical portrait and $U$ be a neighborhood of $\Theta^{\prime}$ in the compact-unlinked topology. Then there exists a finite settled lamination $(\mathcal{L}, g)$ with critical classes $\mathbf{c}$ and $\mathbf{d}$ such that, if $\Theta^{\prime \prime}$ is the critical portrait of a lamination containing an order isomorphic copy of $(\mathcal{L}, g)$, then either $\Theta^{\prime \prime} \in U$ or $R_{1 / 2}\left(\Theta^{\prime \prime}\right) \in U$. Moreover, $(\mathcal{L}, g)$ can be continued to a c-lamination. 


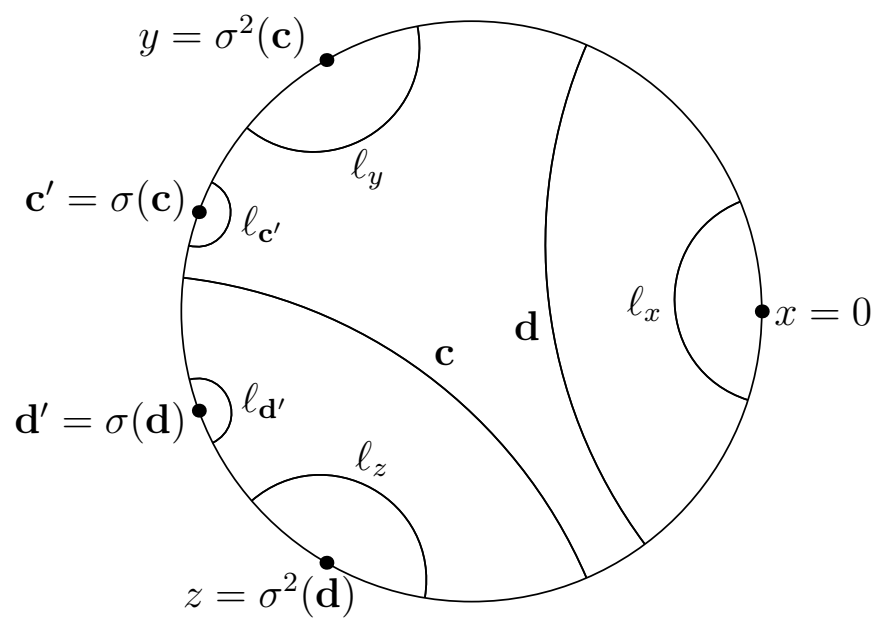

FIGURE 3.2.1. A c-lamination constructed as in the proof of Lemma 3.2.2, with $M=1$ and $N=2$.

Proof. Let $\Theta^{\prime}=\left\{\mathbf{c}^{\prime}, \mathbf{d}^{\prime}\right\}$. There are cases to consider: either $I_{\mathbf{c}^{\prime}}$ contains a fixed point (equivalently $\sigma_{3}\left(\mathbf{c}^{\prime}\right) \notin I_{\mathbf{c}^{\prime}}$ ), or $I_{\mathbf{d}^{\prime}}$ contains a fixed point (equivalently $\left.\sigma_{3}\left(\mathbf{d}^{\prime}\right) \notin I_{\mathbf{d}^{\prime}}\right)$, or $I_{\mathbf{c}^{\prime}}$ and $I_{\mathbf{d}^{\prime}}$ each contain a period two point and no fixed point (equivalently $\sigma_{3}\left(\mathbf{c}^{\prime}\right) \in I_{\mathbf{c}}^{\prime}$ and $\left.\sigma_{3}\left(\mathbf{d}^{\prime}\right) \in I_{\mathbf{d}^{\prime}}\right)$. We consider the case that $I_{\mathbf{d}}^{\prime}$ contains 0 ; without loss of generality, the only other case is the third, and we leave its consideration to the reader.

Given $t \in \mathbb{S}^{1}$, let $W(t)=\left(w_{0}(t), w_{1}(t), \ldots\right)$ be a sequence of arcs $(0,1 / 3),(1 / 3,2 / 3),(2 / 3,0)$ or points $0,1 / 3,2 / 3$ such that $\sigma_{3}^{j}(t) \in w_{j}(t)$ for all $j \geq 0$. The initial segment of $W(t)$ of length $k$ is denoted by $W_{k}(t)$.

It is easy to see that there are arbitrarily large numbers $N>M$ and a critical portrait $\Theta=\{\mathbf{c}, \mathbf{d}\}$ with $\mathbf{c}, \mathbf{d}$ disjoint which have the following properties.

(i) The leaves $\mathbf{c}$ and $\mathbf{d}$ are disjoint from $\{k / 12, k=0, \ldots, 11\}$.

(ii) $\left\{\sigma_{3}^{N}(\mathbf{c}), \sigma_{3}^{N}(\mathbf{d})\right\}=\{1 / 3,2 / 3\}$.

(iii) Any critical portrait $\Theta^{\prime \prime}$ with two critical leaves $c^{\prime \prime}, \mathbf{d}^{\prime \prime}$ such that $W_{M}\left(\mathbf{d}^{\prime \prime}\right)=W_{M}(\mathbf{d})$ and $W_{M}\left(\mathbf{c}^{\prime \prime}\right)=W_{M}(\mathbf{c})$ belongs to $U$.

From now on we fix $\Theta$. Since the endpoints of $\Theta$ are strictly preperiodic, Theorem 1.2.5 implies that $\sim=\sim_{\Theta}$ (defined in Definition 1.2.4 has several properties. In particular, $\{0\}=\mathbf{x},\left\{\sigma_{3}^{N}(\mathbf{d})\right\}=\mathbf{y}$ and $\left\{\sigma_{3}^{N}(\mathbf{c})\right\}=\mathbf{z}$ are 〜-buds.

Also, $\mathbf{c}$ and $\mathbf{d}$ are $\sim$-classes. To see this, suppose $\mathrm{g}$ is the $\sim$-class containing c. Since $\sigma_{3}^{N}(\mathbf{c})=\mathbf{z}$ is a bud, we have that $\sigma^{N}(\mathbf{g})=\mathbf{z}$, so $\mathbf{g}$ is finite and non-periodic. If $\sigma_{3}(\mathbf{g})$ is not degenerate, then it is at some point critical, and hence contains $\mathbf{d}$. We then see that $\mathbf{g}$ eventually maps to $\sigma_{3}^{N}(\mathbf{d})=2 / 3$, but this is a contradiction, as neither $1 / 3$ nor $2 / 3$ ever maps to the other. 
We will now define the required settled lamination by identifying a finite sublamination of the lamination $\sim$. Thus, in what follows we will consider preimages and pullbacks of leaves in the sense of the lamination $\sim$. Let $\mathbf{d}_{-1}, \ldots, \mathbf{d}_{-N}$ denote repeated preimages of $\mathbf{d}$ into $A_{\mathbf{d}}$. Note then that $0 \in$ $I_{\mathbf{d}_{-N}} \subset \ldots \subset I_{\mathbf{d}_{-1}} \subset A_{\mathbf{d}}$. It is also apparent that 0 is the only point of the forward orbits of $\mathbf{c}$ and $\mathbf{d}$ which lies in $I_{\mathbf{d}_{-N}}$ (any forward image of $\mathbf{c}$ or $\mathbf{d}$ maps to 0 in at most $N$ steps and no point in $I_{\mathbf{d}_{-N}}$ does so except for 0 ). We set $\ell_{x}=\mathbf{d}_{-N}$ and choose preimages $\ell_{y}$ and $\ell_{z}$ of $\ell_{x}$ to satisfy $\mathbf{y} \in I_{\ell_{y}}$, $\mathbf{z} \in I_{\ell_{z}}$.

Let us show that $\ell_{y}$ is contained in one component of the $\Theta$-unlinked class $Q$ containing $\mathbf{y}$. Indeed, by way of contradiction suppose that $\ell_{y}$ intersects two components of $Q$. Then $\ell_{y}$ separates $\mathbf{c}$ from $\mathbf{d}$. Hence $\sigma_{3}\left(\ell_{y}\right)=\ell_{x}$ separates $\sigma_{3}(\mathbf{c})$ from $\sigma_{3}(\mathbf{d})$. Therefore, $I_{\ell_{x}}$ contains either $\sigma_{3}(\mathbf{c})$ or $\sigma_{3}(\mathbf{d})$, a contradiction. Moreover, no point of the forward orbits of $\mathbf{c}$ and $\mathbf{d}$ enters $I_{\ell_{y}} \cup I_{\ell_{z}}$ except for $\mathbf{y}$ and $\mathbf{z}$.

Since $\sigma_{3}^{N+1}(\mathbf{c})=\sigma_{3}^{N+1}(\mathbf{d})=0$ and $N>M$, we can pull back the leaves $\ell_{y}$ and $\ell_{z}$ along the branches of the backward orbit of 0 towards $\sigma_{3}^{M}(\mathbf{d})$ and $\sigma_{3}^{M}(\mathbf{c})$ respectively. Denote by $I\left(\sigma_{3}^{M+i}(\mathbf{d})\right)$ the pullback of $I_{\ell_{y}}$ which contains the point $\sigma_{3}^{M+i}(\mathbf{d})$, and by $\ell\left(\sigma_{3}^{M+i}(\mathbf{d})\right)$ the $\sim$-leaf which has the same endpoints as $I\left(\sigma_{3}^{M+i}(\mathbf{d})\right)$. Observe that the only point of the orbits of $\mathbf{c}, \mathbf{d}$ in $I\left(\sigma_{3}^{M+i}(\mathbf{d})\right)$ is $\sigma_{3}^{M+i}(\mathbf{d})$. Similarly, denote by $I\left(\sigma_{3}^{M+i}(\mathbf{c})\right)$ the pullback of $I_{\ell_{z}}$ which contains the point $\sigma_{3}^{M+i}(\mathbf{c})$, and by $\ell\left(\sigma_{3}^{M+i}(\mathbf{c})\right)$ the $\sim$ leaf which has the same endpoints as $I\left(\sigma_{3}^{M+i}(\mathbf{c})\right)$. Here $0 \leq i \leq N-M$ so that $I\left(\ell_{y}\right)=I\left(\sigma_{3}^{N}(\mathbf{d})\right)=I(y), I\left(\ell_{z}\right)=I\left(\sigma_{3}^{N}(\mathbf{c})\right)=I(z)$. Put $I(x)=I_{\ell_{x}}$.

Set

$$
\begin{aligned}
& V=I\left(\sigma_{3}^{M}(\mathbf{d})\right) \cup I\left(\sigma_{3}^{M+1}(\mathbf{d})\right) \cup \ldots \cup I(y) \cup \\
& I\left(\sigma_{3}^{M}(\mathbf{c})\right) \cup I\left(\sigma_{3}^{M+1}(\mathbf{c})\right) \cup \ldots \cup I(z) \cup I(x)
\end{aligned}
$$

Note that all the intervals comprising this union are pairwise disjoint. Consider the critical lamination $\mathcal{L}$ defined as

$$
\mathcal{L}=\left\{\sigma_{3}^{i}(\mathbf{c}), \sigma_{3}^{i}(\mathbf{d})\right\}_{i=0}^{M} \cup\left\{\mathbf{d}_{-j}\right\}_{j=1}^{N} \cup\left\{\ell\left(\sigma_{3}^{M+i}(\mathbf{c})\right), \ell\left(\sigma_{3}^{M+i}(\mathbf{d})\right)\right\}_{i=0}^{N-M} .
$$

Define $\widehat{\mathcal{L}}$ as $\mathcal{L} \backslash\left\{\sigma_{3}^{M}(\mathbf{c}), \sigma_{3}^{M}(\mathbf{d})\right\}$ and set $g=\left.\sigma_{3}\right|_{\widehat{\mathcal{L}}}$. This defines the settled dynamical lamination $(\mathcal{L}, g)$ whose only last classes are $\sigma_{3}^{M}(\mathbf{c})=$ $\mathbf{c}^{\prime}, \sigma_{3}^{M}(\mathbf{d})=\mathbf{d}^{\prime}$. Moreover, by construction $\ell\left(\mathbf{c}^{\prime}\right)$ is a preimage of $\mathbf{d}$ which is adjacent to $\mathbf{c}^{\prime}$ in $(\mathcal{L}, g)$ and which maps to $\mathbf{d}_{-N}$ by $\sigma_{3}^{N+1-M}$ and then to d by $\sigma_{3}^{2 N+1-M}$.

Consider a lamination $\sim$ with critical portrait $\Theta^{\prime \prime}$ containing an order isomorphic copy of $(\mathcal{L}, g)$. We need to show that then $\Theta^{\prime \prime} \in U$ or $R_{1 / 2}\left(\Theta^{\prime \prime}\right) \in$ $U$. Set $\Theta^{\prime \prime}=\left\{\mathbf{c}^{\prime \prime}, \mathbf{d}^{\prime \prime}\right\}$. Let $h$ be the order isomorphism between $(\mathcal{L}, g)$ 
and the appropriate finite sublamination $\left(\mathcal{L}^{\prime \prime},\left.\sigma_{3}\right|_{\widehat{\mathcal{L}}^{\prime \prime}}\right)$ of $\mathcal{L}_{\sim}$ so that $\mathbf{d}^{\prime \prime}=$ $h(\mathbf{d}), \mathbf{c}^{\prime \prime}=h(\mathbf{c})$. The dynamics of $g$ (and hence of $\sigma_{3}$ on $\mathcal{L}^{\prime \prime}$ ) implies that there is a $\sigma_{3}$-fixed point in $h(I(x))$; without loss of generality we may assume that this fixed point is 0 (otherwise we will apply $R_{1 / 2}$ to $\sim$ ). Moreover, $h(I(y))$ must contain one $\sigma_{3}$-preimage of 0 not equal to 0 while $h(I(z))$ contains the other $\sigma_{3}$-preimage of 0 not equal to 0 . Since $h$ is an order isomorphism and $g=\left.\sigma_{3}\right|_{\widehat{\mathcal{L}}}$, it follows that in fact $I(y)$ contains the same preimage of 0 as $h(I(y))$, and $I(z)$ contains the same preimage of 0 as $h(I(z))$.

Now, the fact that $(\mathcal{L}, g)$ and $\left(\mathcal{L}^{\prime \prime},\left.\sigma_{3}\right|_{\widehat{\mathcal{L}}^{\prime \prime}}\right)$ are order isomorphic implies that $W_{M}(\mathbf{c})=W_{M}\left(\mathbf{c}^{\prime \prime}\right)$ and $W_{M}(\mathbf{d})=W_{M}\left(\mathbf{d}^{\prime \prime}\right)$. By the choice of $M$ this implies that $\Theta^{\prime \prime} \in U$ as desired.

Finally, we note that it is possible to continue $(\mathcal{L}, g)$ to a c-lamination. Indeed, $\mathbf{c}^{\prime}$ and $\mathbf{d}^{\prime}$ are both adjacent to preimages of $\mathbf{d}$. Moreover, it follows from the above that the arc under $\ell\left(\mathbf{d}^{\prime}\right)$ maps onto the entire $\mathbb{S}^{1}$ in the oneto-one fashion (except for its endpoints) by $\sigma_{3}^{2 N+2-M}$. This allows us to find the $\sigma_{3}^{2 N+2-M}$-preimage $\mathbf{m}$ of $\mathbf{c}$ adjacent to $\mathbf{d}^{\prime}$ which does not map by $\sigma_{3}$ under the leaf $\boldsymbol{\ell}$ adjacent to $\mathbf{c}^{\prime}$ until it $(\mathbf{m})$ maps to $\mathbf{c}$ and then to $\mathbf{c}^{\prime}$. One then adds a triple $\mathbf{T}_{1}$ leaf-like and adjacent to both $\mathbf{d}^{\prime}$ and to $\mathbf{m}$ very close to $\mathbf{m}$ so that the appropriate initial segment of the orbit of $\mathbf{T}_{1}$ follows the orbit of $\mathbf{m}$ until $\mathbf{m}$ maps to $\mathbf{c}$ and then to $\mathbf{c}^{\prime}$. At this moment the image of $\mathbf{T}_{1}$ is leaf-like adjacent to $\mathbf{c}^{\prime}$ and to $\ell$. The result is a $\mathbf{c}$-lamination.

We can now combine all of these ingredients to give a proof of our main result. Recall that $\mathcal{W} \mathcal{T}_{3}$ is the family of all cubic critical WT-portraits.

Main theorem. For each open $U \subset \mathcal{A}_{3}$ there is an uncountable set $\mathcal{B} \subset$ $U \cap \mathcal{A P}_{3} \cap \mathcal{W} \mathcal{T}_{3}$ of critical portraits $\Theta$ such that the following facts hold:

(1) there exists a wandering branch point in $J_{\sim_{\Theta}}$ whose orbit is condense in $J_{\sim_{\Theta}}$;

(2) all maps $\left.f_{\sim_{\Theta}}\right|_{J_{\sim_{\Theta}}}, \Theta \in \mathcal{B}$, are pairwise non-conjugate;

(3) for each $\Theta \in \mathcal{B}$ there exists a polynomial $P_{\Theta}$ such that $\left.P_{\Theta}\right|_{J_{P_{\Theta}}}$ is conjugate to $f_{\sim_{\Theta}} \mid J_{\sim_{\Theta}}$.

Proof. Fix an open set $U \subset \mathcal{A}_{3}$ and full sequences of precritical itineraries $\left(\tau_{n}^{\mathbf{c}}\right)_{n=1}^{\infty},\left(\tau_{n}^{\mathbf{d}}\right)_{n=1}^{\infty}$ ending in $\mathbf{c}$ and $\mathbf{d}$ respectively. By Lemma 3.2.2 for the neighborhood $U$ there exists a finite settled lamination $\left(\mathcal{L}_{0}, g_{0}\right)$ and a continuation $\left(\mathcal{L}_{0}^{\prime}, g_{0}^{\prime}\right)$ of $\left(\mathcal{L}_{0}, g_{0}\right)$ such that $\left(\mathcal{L}_{0}^{\prime}, g_{0}^{\prime}\right)$ is a c-lamination. Then by Theorem 3.1 .10 there is a $\sigma_{3}$-invariant lamination $\sim$ continuing $\left(\mathcal{L}_{0}^{\prime}, g_{0}^{\prime}\right)$ satisfying (1).

Let now prove claim (2) of the theorem. Let $\hat{\Theta}$ be the critical portrait of $\sim$. Since $\sim$ continues $\left(\mathcal{L}_{0}, g_{0}\right)$, it follows by Lemma 3.2.2 that $\hat{\Theta} \in$ $U \cap \mathcal{A P}_{3} \cap \mathcal{W} \mathcal{T}_{3}$. Consider the set $\hat{\mathcal{B}}$ of all critical WT-portraits constructed 
in this way. It remains to show that there is an uncountable subcollection of $\hat{\mathcal{B}}$ such that the corresponding topological polynomials are pairwise nonconjugate. Recall that the construction of laminations is done on the basis of Lemma 2.3.7 (see the comment after Lemma 2.3.7). Since the images of the points $\sigma_{3}(\hat{\mathbf{c}}), \sigma_{3}(\hat{\mathbf{d}})$ shadow longer and longer segments of the forward orbit of $\hat{\mathbf{T}}_{1}$, then the $\hat{\Theta}$-itineraries of $\hat{\mathbf{c}}$ and $\hat{\mathbf{d}}$ are completely defined by the behavior of the triangle $\hat{\mathbf{T}}_{1}$. On the other hand, the behavior of the triangle $\hat{\mathbf{T}}_{1}$ is completely defined by the sequences of itineraries $\left(\tau_{n}^{\mathbf{c}}\right)_{n=1}^{\infty}$ and $\left(\tau_{n}^{\mathbf{d}}\right)_{n=1}^{\infty}$. Hence the $\hat{\Theta}$-itineraries of $\hat{\mathbf{c}}$ and $\hat{\mathbf{d}}$ are completely determined by by the sequences of itineraries $\left(\tau_{n}^{\mathbf{c}}\right)_{n=1}^{\infty}$ and $\left(\tau_{n}^{\mathbf{d}}\right)_{n=1}^{\infty}$.

Suppose that two pairs $X$ and $Y$ of full sequences of itineraries differ, and denote the critical portraits $\Theta_{X}=\left(\hat{\mathbf{c}}_{X}, \hat{\mathbf{d}}_{X}\right)$ and $\Theta_{Y}=\left(\hat{\mathbf{c}}_{Y}, \hat{\mathbf{d}}_{Y}\right)$ as constructed above. Let us show that the itineraries of $\sigma_{3}\left(\hat{\mathbf{c}}_{X}\right)$ and $\sigma_{3}\left(\hat{\mathbf{c}}_{Y}\right)$ in their respective laminations are distinct. Without loss of generality, suppose that for some integer $n$ the itineraries of $g_{n}^{X}\left(\mathbf{c}_{X}\right)$ and $g_{Y}^{n}\left(\mathbf{c}_{Y}\right)$ coincide until a certain moment when the following holds:

(1) the triangles $\mathbf{T}^{l}\left(\mathcal{L}_{n}^{X}, g_{n}^{X}\right)$ and $\mathbf{T}^{l}\left(\mathcal{L}_{n}^{Y}, g_{n}^{Y}\right)$ are adjacent to $g_{X}^{n}\left(\mathbf{c}_{X}\right)$ and $g_{X}^{n}\left(\mathbf{c}_{Y}\right)$, respectively;

(2) the triangles $\mathbf{T}^{l}\left(\mathcal{L}_{n}^{X}, g_{n}^{X}\right)$ and $\mathbf{T}^{l}\left(\mathcal{L}_{n}^{Y}, g_{n}^{Y}\right)$ are also adjacent to $\ell\left(\mathcal{L}_{n}^{X}, g_{n}^{X}\right)$ and $\ell\left(\mathcal{L}_{n}^{Y}, g_{n}^{Y}\right)$ which they will shadow according to the construction;

(3) the itineraries of $\ell\left(\mathcal{L}_{n}^{X}, g_{n}^{X}\right)$ and $\ell\left(\mathcal{L}_{n}^{Y}, g_{n}^{Y}\right)$ are distinct because they were constructed using distinct itineraries from $X$ and $Y$.

Hence, the itineraries of $\sigma_{3}\left(\hat{\mathbf{c}}_{X}\right)$ and $\sigma_{3}\left(\hat{\mathbf{c}}_{Y}\right)$ are also distinct. This implies that if $X \neq Y$, then $\Theta_{X} \neq \Theta_{Y}$ and the critical itineraries of $\Theta_{X}$ and $\Theta_{Y}$ are distinct. Therefore in any open set in $\mathcal{A}_{3}$ there are uncountably many critical WT-portraits (as there are uncountably many distinct pairs of full sequences of itineraries with each itinerary ending in $\mathbf{c}$ or $\mathbf{d}$ ). In terms of their topological polynomials, $f_{\Theta_{X}}$ and $f_{\Theta_{Y}}$ may yet be conjugate, but can only differ by the labeling of their critical leaves. There is therefore an uncountable subset of critical portraits in $U \cap \mathcal{A}_{3} \cap \mathcal{W} \mathcal{T}_{3}$ whose induced topological polynomials on their topological Julia sets are pairwise nonconjugate. This completes the proof of (2).

To prove (3) observe that by Kiwi's results (see Theorem 1.2.5) for each topological polynomial $f$ constructed above there exists a complex polynomial $P$ which is monotonically semiconjugate to the $f$ on its Julia set. By (1) all these topological polynomials $f$ have points with condense orbits in their topological Julia sets. Therefore by [2, Theorem 3.6(1)] $P$ is conjugate to $f$ as desired. 


\section{REFERENCES}

[1] B. Bielefield, Y. Fisher., J. H. Hubbard, The classification of critically preperiodic polynomials as dynamical systems, Journal AMS 5 (1992), pp. 721-762.

[2] A. Blokh, C. Curry, L. Oversteegen, Density of orbits in laminations and the space of critical portraits, preprint arXiv:1104.4130 (2011), to appear in Discrete and Continuous Dynamical Systems.

[3] A. Blokh, G. Levin, An inequality for laminations, Julia sets and "growing trees", Erg. Th. and Dyn. Sys. 22 (2002), pp. 63-97.

[4] A. Blokh, L. Oversteegen, Wandering gaps for weakly hyperbolic polynomials, in: "Complex dynamics: Families and Friends", ed. by D. Schleicher, A K Peters (2008), pp. 139-168.

[5] B. Branner, J. H. Hubbard, The iteration of cubic polynomials. Part I: The global topology of parameter space, Acta Math. 160 (1988), 143-206.

[6] Y. Fisher, The classification of critically preperiodic polynomials, $\mathrm{PhD}$ Thesis, Cornell University (1989).

[7] L. Goldberg, J. Milnor, Fixed points of polynomial maps II: Fixed point portraits, Ann. Scient. École Norm. Sup., $4^{e}$ série, 26 (1993), 51-98

[8] J. Kiwi, Wandering orbit portraits, Trans. Amer. Math. Soc. 354 (2002), 1473-1485.

[9] J. Kiwi, Real laminations and the topological dynamics of complex polynomials, Advances in Math. 184 (2004), 207-267.

[10] J. Kiwi, Combinatorial continuity in complex polynomial dynamics, Proc. London Math. Soc. 91 (2005), 215-248.

[11] O. Kozlovski, S. van Strien, Local connectivity and quasi-conformal rigidity of nonrenormalizable polynomials, Proc. Lond. Math. Soc. (3) 99 (2009), 275-296.

[12] P. Lavaurs, Systèmes dynamiques holomorphes: explosion de points périodiques, $\mathrm{Phd}$ Thesis, Université Paris-Sud (1989).

[13] J. Milnor, Dynamics in One Complex Variable, Third Edition, Princeton, 2006.

[14] A. Poirier, On postcritically finite polynomials, parts I, II, Stony Brook IMS Preprint 1993-5 and 1993-7 (1993).

[15] A. Poirier, Crtitical portraits for postcritically finite polynomials, Fund. Math. 203, \#2 (2009), 107-163.

[16] A. Poirier, Hubbard trees, Fund. Math. 208, \#3 (2010), 193-248.

[17] W. Thurston. The combinatorics of iterated rational maps (1985), in: "Complex dynamics: Families and Friends", ed. by D. Schleicher, A K Peters (2008), pp. 3-137.

(Alexander Blokh, Lex Oversteegen and Clinton Curry) Department of Mathematics, University of Alabama at Birmingham, Birmingham, AL 352941170, TEL.: (205)934-2154, FAX: (205)934-9025

E-mail address, Alexander Blokh: ablokh@math.uab.edu

E-mail address, Lex Oversteegen: overstee@math.uab.edu

E-mail address, Clinton Curry: clintonc@math. sunysb.edu 